\title{
Symmetrization of a Family of Cauchy-Like Kernels: Global Instability
}

\section{Loredana Lanzani $^{1} \cdot$ Malabika Pramanik $^{2}$}

Received: 24 February 2021 / Revised: 16 August 2021 / Accepted: 21 September 2021 /

Published online: 5 January 2022

(c) The Author(s), under exclusive licence to Springer Science+Business Media LLC, part of Springer Nature 2022

\section{Abstract}

The fundamental role of the Cauchy transform in harmonic and complex analysis has led to many different proofs of its $L^{2}$ boundedness. In particular, a famous proof of Melnikov-Verdera (A geometric proof of the $L^{2}$ boundedness of the Cauchy integral on Lipschitz graphs. Int Math Res Not 7:325-331, 1995) relies upon an iconic symmetrization identity of Melnikov (Analytic capacity: a discrete approach and the curvature of measures. Mat Sb 186(6):57-76, 1995) linking the universal Cauchy kernel $K_{0}$ to Menger curvature. Analogous identities hold for the real and the imaginary parts of $K_{0}$ as well. Such connections have been immensely productive in the study of singular integral operators and in geometric measure theory. In this article, given any function $h: \mathbb{C} \rightarrow \mathbb{R}$, we consider an inhomogeneous variant $K_{h}$ of $K_{0}$ which is inspired by complex function theory. While an operator with integration kernel $K_{h}$ is easily seen to be $L^{2}$-bounded for all $h$, the symmetrization identities for each of the real and imaginary parts of $K_{h}$ show a striking lack of robustness in terms of boundedness and positivity, two properties that were critical in Melnikov and Verdera (A geometric proof of the $L^{2}$ boundedness of the Cauchy integral on Lipschitz graphs. Int Math Res Not 7:325-331, 1995) and in subsequent works by many authors. Indeed here we show that for any continuous $h$ on $\mathbb{C}$, the only member of $\left\{K_{h}\right\}_{h}$ whose symmetrization has the right properties is $K_{0}$ ! This global instability complements our previous investigation (Lanzani and Pramanik, Symmetrization of a Cauchy-like kernel on curves. J Funct Anal (Preprint). arXiv:2001.09375) of symmetrization identities in the restricted setting of a curve, where a sub-family of $\left\{K_{h}\right\}_{h}$ displays very different behaviour than its global counterparts considered here. Our methods of proof have some overlap with techniques in recent work of Chousionis and Prat, Some Calderòn-Zygmund kernels

Malabika Pramanik

malabika@math.ubc.ca

Loredana Lanzani

llanzani@syr.edu

1 Department of Mathematics, Syracuse University, Syracuse, NY 13244-1150, USA

2 Department of Mathematics, University of British Columbia, Room 121, 1984 Mathematics Rd.,

Vancouver, BC V6T 1Z2, Canada 
and their relation to Wolff capacity. Math Z 282:435-460, 2016) and Chunaev, A new family of singular integral operators whose $L^{2}$ boundedness implies rectifiability. J Geom Anal 27:2725-2757, 2017).

Keywords Cauchy integral · Double layer potential · Menger curvature · Singular integral $\cdot$ Kernel symmetrization

Mathematics Subject Classification $30 \mathrm{C} 40 \cdot 31 \mathrm{~A} 10 \cdot 31 \mathrm{~A} 15$

\section{Introduction}

Multivariate algebraic expressions that are invariant under permutations of the underlying variables are termed symmetric forms. Identities involving such forms, henceforth referred to as symmetrization identities, abound in mathematics. Their appeal lies in the physical interpretation of the various quantities that they embody, which could be geometric, analytic or combinatorial in nature. One such instance [17] was discovered in 1995 by M. Melnikov in regards to the universal Cauchy kernel

$$
K_{0}(w, z):=\frac{1}{w-z}, \quad z, w \in \mathbb{C}, \quad z \neq w
$$

This exceptionally simple identity is the source of many results of import in harmonic and complex analysis, and the starting point of this article. We begin by describing it. For any complex-valued function $K$ defined on a domain in $\mathbb{C}^{2}$, let $S[K]$ denote the following symmetrized form associated with $K$ :

$$
\mathrm{S}[K]:=\sum_{\sigma \in S_{3}} K\left(z_{\sigma(1)}, z_{\sigma(2)}\right) \overline{K\left(z_{\sigma(1)}, z_{\sigma(3)}\right)}
$$

where $S_{3}$ denotes the group of permutations over three elements, and $\mathbf{z}=\left\{z_{1}, z_{2}, z_{3}\right\}$ is any three-tuple of distinct points in $\mathbb{C}$ for which the above expression is meaningful. The symmetrization identity of Melnikov [17] concerns $S\left[K_{0}\right]$. Namely, it says

$$
\mathrm{S}\left[K_{0}\right]=\sum_{\sigma \in S_{3}} \frac{1}{z_{\sigma(1)}-z_{\sigma(2)}} \times \frac{1}{\overline{z_{\sigma(1)}-z_{\sigma(3)}}}=c^{2}(\mathbf{z})
$$

where $c(\mathbf{z})$ represents the Menger curvature for the points $\left\{z_{1}, z_{2}, z_{3}\right\}$. Let us recall that the Menger curvature of three non-collinear points is the reciprocal of the radius of the unique circle that passes through these three points. If the points are collinear, $c(\mathbf{z})$ is taken to be zero. Moreover, the Menger curvature $c^{2}(\mathbf{z})$ splits evenly between the symmetric forms of the real and imaginary parts, in the following sense ${ }^{1}$ :

$$
S\left[K_{0}\right]=S\left[\operatorname{Re}\left(K_{0}\right)\right]+S\left[\operatorname{Im}\left(K_{0}\right)\right] \text {, with }
$$

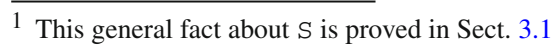




$$
\begin{aligned}
& \mathrm{S}\left[\operatorname{Re}\left(K_{0}\right)\right]=\sum_{\sigma \in S_{3}} \operatorname{Re}\left(\frac{1}{z_{\sigma(1)}-z_{\sigma(2)}}\right) \times \operatorname{Re}\left(\frac{1}{z_{\sigma(1)}-z_{\sigma(3)}}\right)=\frac{1}{2} c^{2}(\mathbf{z}), \\
& \mathrm{S}\left[\operatorname{Im}\left(K_{0}\right)\right]=\sum_{\sigma \in S_{3}} \operatorname{Im}\left(\frac{1}{z_{\sigma(1)}-z_{\sigma(2)}}\right) \times \operatorname{Im}\left(\frac{1}{z_{\sigma(1)}-z_{\sigma(3)}}\right)=\frac{1}{2} c^{2}(\mathbf{z}) .
\end{aligned}
$$

In 1995, Melnikov and Verdera [18] discovered that the identity (1.2) leads to a new proof of famous results by A. Calderòn; Coifman, McIntosh and Meyer that established $L^{2}(\Gamma, \sigma)$-regularity of the Cauchy transform for a planar Lipschitz curve $\Gamma[2,9]$. The new proof [18] and the subsequent work by Mattila, Melnikov and Verdera [16] brought to the fore new connections between the analytic capacity and the rectifiability of the support of the reference measure: thus a hugely prolific line of investigation began that has had a deep and ever-lasting impact on the theory of singular integral operators and on geometric measure theory. A survey of the extensive literature would take us outside of the scope of the present work; instead we defer to the monographs [19] and [22] and, for more recent progress, to [1-8, 10, 11, 13-15, 20, 21, 23].

\subsection{Objectives}

The purpose of this article is to provide analogues of the global symmetrization identities (1.2), (1.3) and (1.4) for a new family of integration kernels that arise naturally in complex function theory and in potential theory. Specifically, we investigate two basic features of such identities,

(i) Global boundedness relative to Menger curvature; and

\section{(i i) Global positivity.}

These properties play an important role in the proofs of many of the results mentioned above and are enjoyed by the symmetrized forms of each of $K_{0}, \operatorname{Re} K_{0}$ and $\operatorname{Im} K_{0}$ following (1.2), (1.3) and (1.4). Here and throughout, by global property we mean a property that holds for all three-tuples $\mathbf{z}=\left\{z_{1}, z_{2}, z_{3}\right\}$ of distinct points in $\mathbb{C}$.

In this article we consider the family of kernels $\left\{K_{h}\right\}$ parametrized by functions $h$ : $\mathbb{C} \rightarrow \mathbb{R}$

$$
K_{h}(w, z):=\frac{\mathrm{e}^{i h(w)}}{w-z}, \quad w, z \in \mathbb{C}, \quad w \neq z .
$$

Setting $h \equiv 0$ (or a constant) yields the universal Cauchy kernel $K_{0}$ (or a constant multiple of it). On the other hand, for non-constant $h$ our kernel $K_{h}$ is significantly different in nature from $K_{0}$ because the latter is a homogeneous function of $w$ and $z$, while the former is not. The definition of $K_{h}$ is driven by complex-analytic considerations that we have discussed in detail in the companion paper [12], where the focus is specialized to those continuous functions $h: \mathbb{C} \rightarrow \mathbb{R}$ such that $j^{*} K_{h}=K_{\Gamma}$ where $j: \Gamma \hookrightarrow \mathbb{C}$ is the inclusion map and

$$
K_{\Gamma}(w, z)=\frac{1}{2 \pi i} \frac{\mathbf{t}_{\Gamma}(w)}{(w-z)}, \quad w, z \in \Gamma, \quad w \neq z
$$


for a given curve $\Gamma$ of class $C^{1}$ with unit tangent vector $\mathbf{t}_{\Gamma}$. By contrast here we work with the unrestricted $K_{h}$ given in (1.5) and we ask the following questions:

Are the symmetrization identities (1.3) and (1.4) preserved by the family $\left\{K_{h}\right\}_{h}$ ? Even if not, do the basic features (i) and (i i) still hold?

We answer both these questions in the negative. Specifically, we show that the exact analogues of the identities (1.3) and (1.4) no longer hold for $\mathrm{S}\left[\operatorname{Re} K_{h}\right]$ or $\mathrm{S}\left[\operatorname{Im} K_{h}\right]$ in the sense that neither is comparable to $c^{2}(\mathbf{z})$. We do obtain identities that serve as substitutes of (1.3) and (1.4), see Proposition 2.2. But these new identities show that neither $S\left[\operatorname{Re} K_{h}\right]$ nor $S\left[\operatorname{Im} K_{h}\right]$ satisfies any of the basic features (i) and (ii). More precisely, in Theorem 2.3 we show that the condition " $h \equiv$ constant on $\mathbb{C}$ " is equivalent to any one of $\mathrm{S}\left[\operatorname{Re} K_{h}\right](\mathbf{z}) / c^{2}(\mathbf{z})$ or $\mathrm{S}\left[\operatorname{Im}\left(K_{h}\right](\mathbf{z}) / c^{2}(\mathbf{z})\right.$ being globally bounded: hence basic feature (i) fails for $S\left[\operatorname{Re} K_{h}\right]$ and $S\left[\operatorname{Im} K_{h}\right]$ with non-constant $h$; in fact it fails already for three-tuples confined to tri-disks, that is $\mathbf{z}=\left(z_{1}, z_{2}, z_{3}\right) \in \mathbb{D}^{3}$ for any disk $\mathbb{D} \subset \mathbb{C}$. On a related theme, in Theorem 2.5 we show that the condition " $h \equiv$ constant" is equivalent to any one of $\mathrm{S}\left[\operatorname{Re} K_{h}\right]$ or $\mathrm{S}\left[\operatorname{Im} K_{h}\right]$ maintaining a fixed sign across $\mathbb{C}^{3}$; thus the basic feature (ii) also fails for $\mathrm{S}\left[\operatorname{Re} K_{h}\right]$ and $\mathrm{S}\left[\operatorname{Im} K_{h}\right]$ whenever $h$ is non-constant.

\subsection{Conclusion}

For nontrivial $h$ the kernels $\left\{\operatorname{Re} K_{h}\right\}$ and $\left\{\operatorname{Im} K_{h}\right\}$ fail to satisfy both of the basic features ( $i$ ) and ( $i$ ) : this is surprising because the symmetrization identity for the full universal Cauchy kernel $K_{0}$, see (1.2), and the corresponding basic features ( $i$ ) and ( $i$ ), hold trivially when $K_{0}$ is replaced by $K_{h}$ on account of the fact that

$$
\mathrm{S}\left[K_{h}\right](\mathbf{z})=\mathrm{S}\left[K_{0}\right](\mathbf{z})
$$

for any $\mathbf{z}$ in $\mathbb{C}^{3}$ and for any $h: \mathbb{C} \rightarrow \mathbb{R}$ (no continuity assumption needed).

This dichotomy stands in contrast with symmetrized identities in the restricted context, and is best appreciated in comparison with the latter class of results. In [12], we study the symmetrized forms $S\left[K_{0}\right], S\left[\operatorname{Re}\left(K_{0}\right)\right]$ and $S\left[\operatorname{Im}\left(K_{0}\right)\right]$ for triples $\mathbf{z}=\left(z_{1}, z_{2}, z_{3}\right) \in$ $\mathbb{C}^{3}$, where each $z_{j}$ lies on a fixed rectifiable curve $\Gamma$ with prescribed regularity. We show that a few members of $\left\{\operatorname{Re} K_{h}\right\}$, namely those for which $j^{*} K_{h}=K_{\Gamma}$ with $K_{\Gamma}$ as in (1.6), will satisfy $\Gamma$-restricted analogues of the basic features (i) and (ii), while their counterparts in $\left\{\operatorname{Im} K_{h}\right\}$ satisfy $\Gamma$-restricted versions of (i) though not necessarily of ( $i$ i).

After completing this work, we learned that some of the methods of proof we employ here bear similarities to techniques developed in unrelated recent work by Chousionis and Prat [5] and by Chunaev [6]. For instance, the proofs of our representation formulæ for $\mathrm{S}\left[\operatorname{Re} K_{h}\right]$ or $\mathrm{S}\left[\operatorname{Im} K_{h}\right]$, given in Proposition 2.2 below, rely upon a certain labeling scheme of the vertices of a triangle, which is a device that also appears in [5,Proposition 3.1] and [6,Lemma 6]; see also Figs. 3 and 4 in [6,p. 2738] and the computations accompanying those diagrams. 


\subsection{Organization of this Paper}

For the reader's convenience, the well-known background is summarized in Sect. 3. In Sects. 2.1 through 2.3 we state the results pertaining to $\operatorname{Re} K_{h}$ and $\operatorname{Im} K_{h}$.

Further results pertaining to $K_{h}^{*}$ (the dual kernel of $K_{h}$ ) are stated in Sect. 2.4. All proofs are collected in Sect. 4.

\section{Description of the Results}

\subsection{Setup}

As mentioned earlier, our kernel $K_{h}$ is given by

$$
K_{h}(w, z):=\frac{\mathrm{e}^{i h(w)}}{w-z},
$$

where $h: \mathbb{C} \rightarrow \mathbb{R}$ is a given function. If $h$ is a constant function, then $K_{h}$ is a constant multiple of $K_{0}$ given in (1.1).

\subsection{Symmetrization Identities for Arbitrary $h$.}

It is immediate to see that

$$
\mathrm{S}\left[K_{h}\right](\mathbf{z})=\mathrm{S}\left[K_{0}\right](\mathbf{z})=c^{2}(\mathbf{z})
$$

for any three-tuple of distinct points and for any $h: \mathbb{C} \rightarrow \mathbb{R}$ (no continuity assumption needed here).

The first natural question that presents itself is whether the phenomenon (2.2) is inherited by the real and the imaginary parts of $K_{h}$. On account of (1.3) and (1.4) this amounts to asking whether the identities

$$
\begin{aligned}
& \mathrm{S}\left[\operatorname{Re} K_{h}\right](\mathbf{z})=\mathrm{S}\left[\operatorname{Re} K_{0}\right](\mathbf{z})=\frac{1}{2} c^{2}(\mathbf{z}), \text { and } \\
& \mathrm{S}\left[\operatorname{Im} K_{h}\right](\mathbf{z})=\mathrm{S}\left[\operatorname{Im} K_{0}\right](\mathbf{z})=\frac{1}{2} c^{2}(\mathbf{z})
\end{aligned}
$$

are true for all (or for some) non-constant $h: \mathbb{C} \rightarrow \mathbb{R}$. We answer this question in the negative.

In order to state the precise result we adopt a specific labeling scheme for three-tuples of non-collinear points.

Definition 2.1 We say that an ordered three-tuple of non-collinear points $(a, b, c)$ is arranged in admissible order (or is admissible, for short) if (a) the orthogonal projection of $c$ onto the line determined by $a$ and $b$ falls in the interior of the line 
segment joining $a$ and $b$, and $(\mathrm{b})$ the triangle $\Delta(a, b, c)$ has positive counterclockwise orientation.

We will show in Sect. 3.3 that any three-tuple of non-collinear points has at least one admissible ordering.

Proposition 2.2 For any non-constant $h: \mathbb{C} \rightarrow \mathbb{R}$ and for any three-tuple $z$ of noncollinear points in $\mathbb{C}$ we have

$$
\begin{aligned}
& S\left[\operatorname{Re} K_{h}\right](z)=c^{2}(z)\left(\frac{1}{2}+\mathcal{R}_{h}(z)\right), \\
& S\left[\operatorname{Im} K_{h}\right](z)=c^{2}(z)\left(\frac{1}{2}-\mathcal{R}_{h}(\boldsymbol{z})\right),
\end{aligned}
$$

where $\mathcal{R}_{h}$ is non-constant and invariant under the permutations of the elements of $z$. If $z=\left(z_{1}, z_{2}, z_{3}\right)$ is admissible then $\mathcal{R}_{h}(z)$ is represented as follows:

$$
\begin{aligned}
\mathcal{R}_{h}(\boldsymbol{z})= & \frac{2 \ell_{1} \ell_{2} \ell_{3}}{(4 \operatorname{Area} \Delta(z))^{2}} \times\left[\ell_{1} \cos \left(\mathfrak{h}_{z_{1}, z_{2}}\left(z_{1}\right)-\theta_{1}\right)+\right. \\
& \left.+\ell_{2} \cos \left(\mathfrak{h}_{z_{1}, z_{2}}\left(z_{2}\right)+\theta_{2}\right)-\ell_{3} \cos \left(\mathfrak{h}_{z_{1}, z_{2}}\left(z_{3}\right)+\theta_{2}-\theta_{1}\right)\right] .
\end{aligned}
$$

Here $\theta_{j}$ denotes the angle at $z_{j}$, and $\ell_{j}$ denotes the length of the side opposite to $z_{j}$ in $\Delta(z)$. Also, we have set

$$
\mathfrak{h}_{z_{1}, z_{2}}(z):=2 h(z)-2 \alpha_{21}, \quad z \in \mathbb{C}
$$

where $\alpha_{21}$ is the principal argument of $z_{2}-z_{1}$ (in an arbitrarily fixed coordinate system for $\mathbb{R}^{2}$ ).

\section{Remarks (A).}

1. A discussion of the invariance properties of these symmetrized forms is in order. While $S\left[K_{0}\right](\mathbf{z})$ (and thus $S\left[K_{h}\right](\mathbf{z})$ ), $S\left[\operatorname{Re} K_{0}\right](\mathbf{z})$ and $S\left[\operatorname{Im} K_{0}\right](\mathbf{z})$ ) are clearly invariant under rotations and translations of $\mathbb{R}^{2}$, this is no longer the case for $\mathrm{S}\left[\operatorname{Re} K_{h}\right](\mathbf{z})$ or $\mathrm{S}\left[\operatorname{Im} K_{h}\right](\mathbf{z})$. Yet, (2.4) and (2.5) show that each of these symmetrized forms contains a term that is invariant under rotations and translations of $\mathbb{R}^{2}$.

2. The well-posedness of the righthand sides of (2.4) and (2.5) for three-tuples of distinct points, as opposed to non-collinear points, is addressed in Sect. 4.1, see Remark (B) in that section.

\subsection{Boundedness and Positivity: Global Results for Arbitrary $h$}

We next ask whether either $\mathrm{S}\left[\operatorname{Re} K_{h}\right](\mathbf{z})$ or $\mathrm{S}\left[\operatorname{Im} K_{h}\right](\mathbf{z})$ obeys the basic global features (i) and (ii). The answer is negative in all instances: we prove below that $\mathcal{R}_{h}$ is either constant (in fact zero) or unbounded! 
Theorem 2.3 Suppose that $h: \mathbb{C} \rightarrow \mathbb{R}$ is continuous. The following are equivalent:

(i) There is a constant $C<\infty$, possibly depending on $h$, such that

$$
\left|\mathcal{R}_{h}(z)\right| \leq C
$$

for any three-tuple $z=\left\{z_{1}, z_{2}, z_{3}\right\}$ of non-collinear points in $\mathbb{C}$.

(ii) $\quad \mathcal{R}_{h}(z)=0$ for any three-tuple of non-collinear points in $\mathbb{C}$.

(iii) $h$ is constant.

The proof will in fact show the following stronger conclusion: if we only assume that $h$ is continuous on a disc $\mathbb{D}_{0} \subset \mathbb{C}$, then the boundedness of $\mathcal{R}_{h}(\mathbf{z})$ for any three-tuple $\mathbf{z}$ of points in $\mathbb{D}_{0}$ is equivalent to $h$ being constant on $\mathbb{D}_{0}$.

An immediate consequence of Theorem 2.3 is that no analog of the positivity condition (i i ) can hold simultaneously for $\mathrm{S}\left[\operatorname{Re} K_{h}\right]$ and $\mathrm{S}\left[\operatorname{Im} K_{h}\right]$ unless $h=$ const. Specifically, we have

Corollary 2.4 Suppose that $h: \mathbb{C} \rightarrow \mathbb{R}$ is continuous. Then

$$
h \text { is constant } \Longleftrightarrow\left(\frac{1}{2}-\mathcal{R}_{h}(z)\right)\left(\frac{1}{2}+\mathcal{R}_{h}(z)\right)>0
$$

for any three-tuple of non-collinear points in $\mathbb{C}$.

In fact more is true.

Theorem 2.5 Suppose that $h: \mathbb{C} \rightarrow \mathbb{R}$ is continuous.

(a) If $h$ is constant, then

$$
\frac{1}{2}+\mathcal{R}_{h}(z)>0
$$

for any three-tuple of non-collinear points.

(b) If $h$ is not constant, then the function

$$
z \mapsto \frac{1}{2}+\mathcal{R}_{h}(z)
$$

changes sign. That is, there exist two three-tuples of non-collinear points $z$ and $z^{\prime}$ such that

$$
\frac{1}{2}+\mathcal{R}_{h}(z)>0 \text { and } \frac{1}{2}+\mathcal{R}_{h}\left(z^{\prime}\right)<0
$$

Furthermore, (a) and (b) are also true with $\frac{1}{2}-\mathcal{R}_{h}$ in place of $\frac{1}{2}+\mathcal{R}_{h}$.

Corollary 2.6 Suppose that $h: \mathbb{C} \rightarrow \mathbb{R}$ is continuous. Then

(a) $h$ is constant $\Longleftrightarrow \frac{1}{2}+\mathcal{R}_{h}(z)>0$ for all three-tuples of non-collinear points.

(b) $h$ is constant $\Longleftrightarrow \frac{1}{2}-\mathcal{R}_{h}(z)>0$ for all three-tuples of non-collinear points. 


\subsection{Further Results}

As is well-known, the Cauchy transform is essentially self-adjoint. In fact if $\mathfrak{C}_{0}^{*}$ denotes the formal $L^{2}$-adjoint of $\mathfrak{C}_{0}$, then the "dual" kernel of $K_{0}$ (which is the kernel for $\mathfrak{C}_{0}^{*}$ ) is

$$
K_{0}^{*}(w, z)=-\overline{K_{0}(w, z)}
$$

giving

$$
\mathrm{S}\left[K_{0}^{*}\right](\mathbf{z})=\mathrm{S}\left[K_{0}\right](\mathbf{z}) .
$$

Thus

$$
\mathrm{S}\left[K_{0}^{*}\right](\mathbf{z})=c^{2}(\mathbf{z})
$$

and the symmetrization estimates for $K_{0}^{*}$ are synonymous with those for $K_{0}$.

We define the dual kernel of $K_{h}(w, z)$ as

$$
K_{h}^{*}(w, z)=\overline{K_{h}(z, w)}
$$

Thus

$$
K_{h}^{*}(w, z)=\frac{\mathrm{e}^{-i h(z)}}{\bar{z}-\bar{w}} .
$$

As the name suggests, if $K_{h}$ is the integration kernel of an integral operator, then $K_{h}^{*}$ is the kernel of its formal adjoint.

In great contrast with (2.7) and (2.8), for non-constant $h$ the symmetrization identities and global estimates for $K_{h}^{*}$ turn out to be very different from those for $K_{h}$. While not directly related to the sequel [12] of this paper, these results are of independent interest and we state them below.

Proposition 2.7 For any non-constant $h: \mathbb{C} \rightarrow \mathbb{R}$ and for any three-tuple $z$ of noncollinear points in $\mathbb{C}$ we have

$$
S\left[K_{h}^{*}\right](z)=c^{2}(\boldsymbol{z}) \mathcal{H}(\boldsymbol{z})
$$

where $\mathcal{H}(z)$ is a non-constant function of $z$ that is invariant under the permutations of the elements of $z$. In particular, if $z=\left(z_{1}, z_{2}, z_{3}\right)$ is admissible then $\mathcal{H}(\boldsymbol{z})$ has the following representation.

$$
\begin{aligned}
\mathcal{H}(z)= & \frac{2 \ell_{1} \ell_{2} \ell_{3}}{(4 \operatorname{Area} \Delta(z))^{2}} \times\left[\ell_{1} \cos \left(h\left(z_{2}\right)-h\left(z_{3}\right)+\theta_{1}\right)+\right. \\
& \left.+\ell_{2} \cos \left(h\left(z_{1}\right)-h\left(z_{3}\right)-\theta_{2}\right)+\ell_{3} \cos \left(h\left(z_{1}\right)-h\left(z_{2}\right)+\theta_{3}\right)\right] .
\end{aligned}
$$

Here $\theta_{j}$ and $\ell_{j}$ are as in the statement of Proposition 2.2.

Theorem 2.8 Suppose that $h: \mathbb{C} \rightarrow \mathbb{R}$ is continuous. The following are equivalent: 
(i) There is a constant $C<\infty$, possibly depending on $h$, such that

$$
|\mathcal{H}(z)| \leq C
$$

for any three-tuple $z=\left\{z_{1}, z_{2}, z_{3}\right\}$ of non-collinear points in $\mathbb{C}$.

(ii) $\mathcal{H}(z)=1$ for any three-tuple of non-collinear points in $\mathbb{C}$.

(iii) $h$ is constant.

\section{Background}

Here we list without proof a few elementary computational tools and basic facts.

\subsection{Basic Properties of Symmetrized Forms}

Recall that the symmetrized form considered in [12] is

$$
\mathrm{S}[K](\mathbf{z}):=\sum_{\sigma \in S_{3}} K\left(z_{\sigma(1)}, z_{\sigma(2)}\right) \overline{K\left(z_{\sigma(1)}, z_{\sigma(3)}\right)}
$$

where $S_{3}$ is the set of all permutations of $\{1,2,3\}$ and $\mathbf{z}$ is any three-tuple of points in $\mathbb{C}$ for which the above expression is meaningful.

- It is easy to see that the above can be equivalently expressed as

$$
\mathrm{S}[K](\mathbf{z})=2 \sum_{\sigma \in S_{3}^{\prime}} \operatorname{Re}\left(K\left(z_{\sigma(1)}, z_{\sigma(2)}\right) \overline{K\left(z_{\sigma(1)}, z_{\sigma(3)}\right)}\right),
$$

where $S_{3}^{\prime}=\{123,213,312\}$.

- It follows that $S[K](\mathbf{z})$ is real-valued and that

$$
\mathrm{S}[\bar{K}](\mathbf{z})=\overline{\mathrm{S}[K](\mathbf{z})}=\mathrm{S}[K](\mathbf{z}) .
$$

- The symmetrized form of $K(w, z)$ can also be expressed as

$$
\mathrm{S}[K](\mathbf{z})=2 \sum_{\substack{j \\ k<l}} \operatorname{Re}\left(K\left(z_{j}, z_{k}\right) \overline{K\left(z_{j}, z_{\ell}\right)}\right),
$$

where it is understood that $j \in\{1,2,3\}$ and for each fixed $j$, the remaining labels $k, \ell \in\{1,2,3\}$ are displayed so that $k<\ell$.

- In particular, if $A(w, z)$ is real-valued, then we have

$$
\mathrm{S}[A](\mathbf{z})=2 \sum_{\substack{j \\ k<l}} A\left(z_{j}, z_{k}\right) A\left(z_{j}, z_{\ell}\right)
$$


- The operation $K \mapsto S[K]$ is not linear:

$$
\mathrm{S}[K+H] \neq \mathrm{S}[K]+\mathrm{S}[H] \text { and } \mathrm{S}[a K] \neq a \mathrm{~S}[K] \text {. }
$$

However if the kernels $A$ and $B$ are real-valued then

$$
\mathrm{S}[A \pm i B]=\mathrm{S}[A]+\mathrm{S}[B]
$$

Thus

$$
S[K]=S[\operatorname{Re} K]+\mathrm{S}[\operatorname{Im} K] .
$$

- For the kernels $\left\{K_{h}\right\}_{h}$ defined in [12], whenever convenient we will write

$$
K_{h}(w, z)=\frac{\mathrm{e}^{i h(w)}(\bar{w}-\bar{z})}{|w-z|^{2}}
$$

and in particular

$$
\operatorname{Re} K_{h}(w, z)=\frac{\operatorname{Re}\left(\mathrm{e}^{-i h(w)}(w-z)\right)}{|w-z|^{2}}
$$

with a corresponding identity for the imaginary part.

- Finally, we point out that for the kernels $K_{h}$ we have

$$
\mathrm{S}\left[K_{h}\right](\mathbf{z})=\mathrm{S}\left[K_{h+c}\right](\mathbf{z}) \text { for any constant } c \in \mathbb{R} \text {. }
$$

\subsection{A Brief Review of Menger Curvature}

The Menger curvature associated to any three element set $\{a, b, c\}$ of distinct points in $\mathbb{C}$, denoted $c(\mathbf{z})$, is the reciprocal of the radius of the circle passing through those points (with the understanding that if the points are collinear, then $c(\mathbf{z})=0$ ). Suppose now that the three points are not collinear and consider the triangle $\Delta(\mathbf{z})$ with vertices $\{a, b, c\}$, which we describe as follows: for each $j \in\{a, b, c\}$ we denote the angle at $j$ by $\theta_{j}$, while $\ell_{j}$ denotes the length of the side opposite to $j$, that is: $\ell_{j}=\left|z_{l}-z_{k}\right|$ where $\{j, k, l\}=\{a, b, c\}$. See Fig. 1 .

We recall a few basic identities that relate $c(\mathbf{z})$ and $\Delta(\mathbf{z})$, see e.g. [24]:

$$
\begin{aligned}
& c(\mathbf{z})=2 \frac{\sin \theta_{j}}{\ell_{j}}, \quad j \in\{a, b, c\}, \quad \text { so in particular } \\
& \text { (Law of sines) } \frac{\sin \theta_{a}}{\ell_{a}}=\frac{\sin \theta_{b}}{\ell_{b}}=\frac{\sin \theta_{c}}{\ell_{c}} ; \\
& 2 \frac{\cos \theta_{j}}{\ell_{k} \ell_{l}}=c^{2}(\mathbf{z}) \frac{\sin 2 \theta_{j}}{4 \sin \theta_{a} \sin \theta_{b} \sin \theta_{c}}, \quad j \in\{a, b, c\}
\end{aligned}
$$


Fig. 1 Labeling system for vertices, side-lengths and angles of a triangle

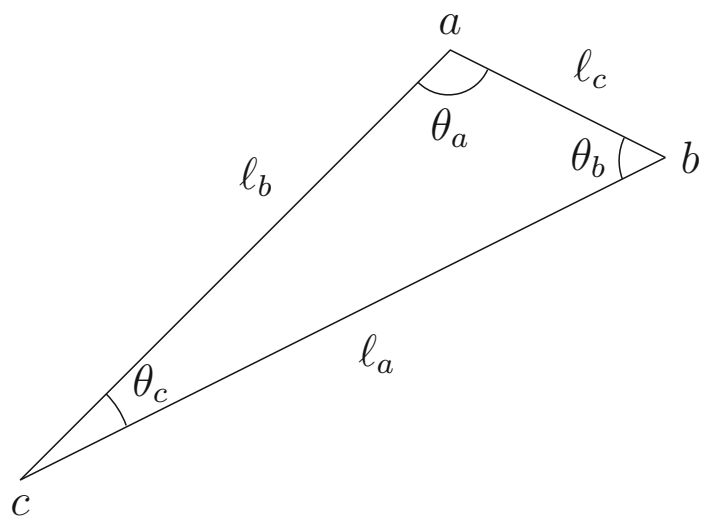

with the understanding that for any given $j=a, b, c$, one takes $l$ and $k$ to be the other two labels in $\{a, b, c\}$, and it follows from (3.11) that

$$
\frac{\cos \theta_{a}}{\ell_{b} \ell_{c}}+\frac{\cos \theta_{b}}{\ell_{a} \ell_{c}}+\frac{\cos \theta_{c}}{\ell_{a} \ell_{b}}=\frac{c^{2}(\mathbf{z})}{2},
$$

because $\sum_{j \in\{a, b, c\}} \sin 2 \theta_{j}=4 \sin \theta_{a} \sin \theta_{b} \sin \theta_{c}$, see again Fig. 1. We also have

$$
c(\mathbf{z})=\frac{4 \operatorname{Area}(\Delta(\mathbf{z}))}{\ell_{a} \ell_{b} \ell_{c}},
$$

which immediately leads us to

$$
c^{2}(\mathbf{z}) \frac{\ell_{a} \ell_{b} \ell_{c}}{(4 \text { Area } \Delta(\mathbf{z}))^{2}}=\frac{1}{\ell_{a} \ell_{b} \ell_{c}} .
$$

\subsection{Labeling Scheme for $\Delta(z)$}

In representing $\mathcal{R}_{h}(\mathbf{z})$, see [12, Proposition 5.2], it will be convenient to re-label the vertices $\{a, b, c\}$ as, say, $\left\{z_{1}, z_{2}, z_{3}\right\}$ where $z_{3}$ is any vertex whose orthogonal projection onto the line determined by the two other vertices falls into the side of $\Delta(\mathbf{z})$ that is opposite to $z_{3}$ and, furthermore, $z_{1}$ and $z_{2}$ are labeled so that the ordered three-tuple $\left(z_{1}, z_{2}, z_{3}\right)$ has positive (counterclockwise) orientation. We also relabel the angles $\theta_{j}$ and the sides $\ell_{j}$ accordingly. We recall from [12] that such labels are called admissible, see Fig. 2. All subsequent formulae appearing in this section refer to admissible ordered three-tuples $\left(z_{1}, z_{2}, z_{3}\right)$.

Since the orthogonal projection of $z_{3}$ onto the line determined by $z_{1}$ and $z_{2}$ occurs at a point $z_{4}$ that lies into the side of $\Delta(\mathbf{z})$ that is opposite to $z_{3}$, we have that

$$
z_{4}=z_{1}+\left(z_{2}-z_{1}\right) \beta=z_{2}+\left(z_{1}-z_{2}\right)(1-\beta) \text { for some } 0<\beta<1 \text {. }
$$



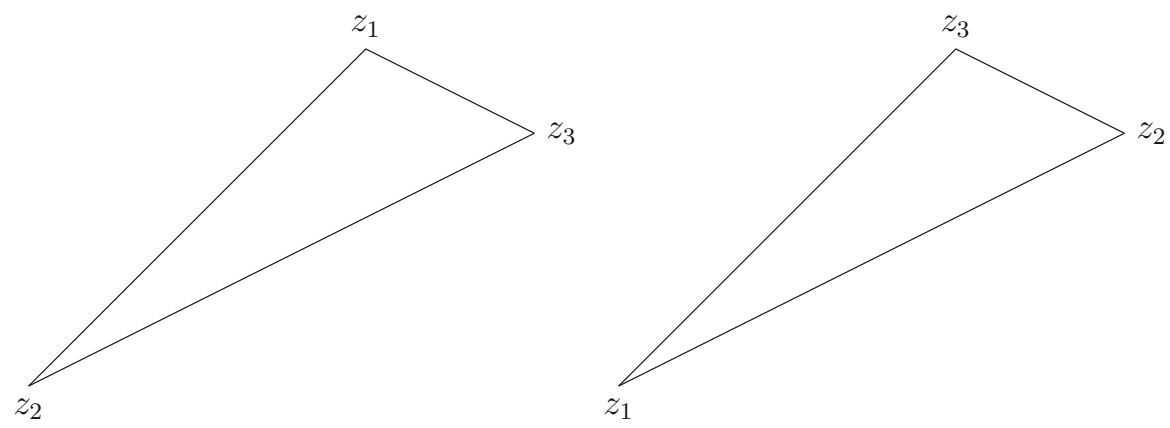

Fig. 2 A triangle in non-admissible form (left), and in admissible form (right)

From these it follows that

$$
z_{3}-z_{4}=i \beta \tan \theta_{1}\left(z_{2}-z_{1}\right)=i(1-\beta) \tan \theta_{2}\left(z_{2}-z_{1}\right),
$$

which in turn grant

$$
z_{2}-z_{3}=\frac{(1-\beta)}{\cos \theta_{2}} \mathrm{e}^{-i \theta_{2}}\left(z_{2}-z_{1}\right)
$$

and

$$
z_{3}-z_{1}=\frac{\beta}{\cos \theta_{1}} \mathrm{e}^{i \theta_{1}}\left(z_{2}-z_{1}\right)
$$

Finally, we recall for future reference that

$$
\ell_{2} \cos \theta_{1}=\left|z_{1}-z_{4}\right|=\beta\left|z_{2}-z_{1}\right|=\beta \ell_{3}, \quad \text { and } \quad \ell_{1} \cos \theta_{2}=(1-\beta) \ell_{3} .
$$

\section{Proofs}

\subsection{Proof of Proposition 2.2}

On account of (3.5) we only need to prove the symmetrization identity for $\operatorname{Re} K_{h}$. To this end we start with

$$
\mathrm{S}\left[\operatorname{Re} K_{h}\right](\mathbf{z})=\frac{2}{\ell_{1}^{2} \ell_{2}^{2} \ell_{3}^{2}} \sum_{\substack{j \\ k<l}} \ell_{j}^{2} \operatorname{Re}\left(\mathrm{e}^{-i h\left(z_{j}\right)}\left(z_{j}-z_{k}\right)\right) \operatorname{Re}\left(\mathrm{e}^{-i h\left(z_{j}\right)}\left(z_{j}-z_{l}\right)\right) .
$$

We expand the sum and again use (3.15) and (3.16) to express $\left(z_{j}-z_{k}\right)$ and $\left(z_{j}-z_{\ell}\right)$ in terms of $\left(z_{2}-z_{1}\right)$. Writing

$$
z_{2}-z_{1}=\ell_{3} \mathrm{e}^{i \alpha_{21}}
$$


we are led to

$$
\begin{aligned}
\mathrm{S}\left[\operatorname{Re} K_{h}\right](\mathbf{z})= & \frac{\beta}{\ell_{2}^{2} \cos \theta_{1}}\left[2 \cos \left(h\left(z_{1}\right)-\alpha_{21}\right) \cos \left(h\left(z_{1}\right)-\alpha_{21}-\theta_{1}\right)\right] \\
& +\frac{(1-\beta)}{\ell_{1}^{2} \cos \theta_{2}}\left[2 \cos \left(h\left(z_{2}\right)-\alpha_{21}\right) \cos \left(h\left(z_{2}\right)-\alpha_{21}+\theta_{2}\right)\right] \\
& +-\ell_{3}^{2} \frac{\beta}{\ell_{2}^{2} \cos \theta_{1}} \frac{(1-\beta)}{\ell_{1}^{2} \cos \theta_{2}}\left[2 \cos \left(h\left(z_{3}\right)-\alpha_{21}-\theta_{1}\right) \cos \left(h\left(z_{3}\right)-\alpha_{21}+\theta_{2}\right)\right] .
\end{aligned}
$$

Applying the formula

$$
2 \cos \gamma \cos \lambda=\cos (\gamma+\lambda)+\cos (\gamma-\lambda)
$$

to each of the three summands (for appropriate choices of $\gamma$ and $\lambda$ ) and recalling our definition $\mathfrak{h}_{z_{1}, z_{2}}(z):=2\left(h(z)-\alpha_{21}\right)$, we obtain

$$
\begin{aligned}
& \mathrm{S}\left[\operatorname{Re} K_{h}\right](\mathbf{z}) \\
& =\frac{\beta}{\ell_{2}^{2} \cos \theta_{1}}\left[\cos \left(\mathfrak{h}_{z_{1}, z_{2}}\left(z_{1}\right)-\theta_{1}\right)+\cos \theta_{1}\right]+\frac{(1-\beta)}{\ell_{1}^{2} \cos \theta_{2}}\left[\cos \left(\mathfrak{h}_{z_{1}, z_{2}}\left(z_{2}\right)+\theta_{2}\right)+\cos \theta_{2}\right] \\
& \quad+-\ell_{3}^{2} \frac{\beta}{\ell_{2}^{2} \cos \theta_{1}} \frac{(1-\beta)}{\ell_{1}^{2} \cos \theta_{2}}\left[\cos \left(\mathfrak{h}_{z_{1}, z_{2}}\left(z_{3}\right)+\theta_{2}-\theta_{1}\right)-\cos \theta_{3}\right] .
\end{aligned}
$$

On account of (3.17), the expression above is reduced to

$$
\begin{aligned}
& \frac{1}{\ell_{2} \ell_{3}}\left[\cos \left(\mathfrak{h}_{z_{1}, z_{2}}\left(z_{1}\right)-\theta_{1}\right)+\cos \theta_{1}\right]+\frac{1}{\ell_{1} \ell_{3}}\left[\cos \left(\mathfrak{h}_{z_{1}, z_{2}}\left(z_{2}\right)+\theta_{2}\right)+\cos \theta_{2}\right]+ \\
& -\frac{1}{\ell_{2} \ell_{1}}\left[\cos \left(\mathfrak{h}_{z_{1}, z_{2}}\left(z_{3}\right)+\theta_{2}-\theta_{1}\right)-\cos \theta_{3}\right] .
\end{aligned}
$$

Applying (3.12) we are led to

$$
\begin{aligned}
S\left[\operatorname{Re} K_{h}\right](\mathbf{z})=\frac{c^{2}(\mathbf{z})}{2} & \\
& +\frac{1}{\ell_{1} \ell_{2} \ell_{3}}\left[\ell_{1} \cos \left(\mathfrak{h}_{z_{1}, z_{2}}\left(z_{1}\right)-\theta_{1}\right)+\ell_{2} \cos \left(\mathfrak{h}_{z_{1}, z_{2}}\left(z_{2}\right)+\theta_{2}\right)\right. \\
& \left.-\ell_{3} \cos \left(\mathfrak{h}_{z_{1}, z_{2}}\left(z_{3}\right)+\theta_{2}-\theta_{1}\right)\right] .
\end{aligned}
$$

The symmetrization identity for $\operatorname{Re} K$ now follows from (3.14). The proof is concluded. A word on the well-posedness of the definition of $\mathcal{R}_{h}(\mathbf{z})$ is in order. If the triangle $\Delta(\mathbf{z})$ has an obtuse or right angle, then $\mathcal{R}_{h}(\mathbf{z})$ is unambiguously defined in the sense that there is a unique admissible form of $\mathbf{z}$ (there is a unique permutation of $\left\{z_{1}, z_{2}, z_{3}\right\}$ that gives the admissible form of $\mathbf{z})$. On the other hand, if $\Delta(\mathbf{z})$ is an acute-angle triangle (all three angles in $\Delta(\mathbf{z})$ are acute) then there are three distinct admissible orderings of $\mathbf{z}$ because $z_{3}$ can be assigned to be any one of the three vertices $a, b$ or $c$. Correspondingly there are three formulations of $\mathcal{R}_{h}(\mathbf{z})$ : these, however, must be 
identical to one another in view of the invariance of $\mathcal{R}_{h}(\mathbf{z})$ under the permutations of $\left\{z_{1}, z_{2}, z_{3}\right\}$. Alternatively, one can directly verify that the three admissible forms of $\mathbf{z}$ lead to the same representation for $\mathcal{R}_{h}(\mathbf{z})$ by invoking the following lemma, whose proof is omitted:

Lemma 4.1 Let $\Delta(z)$ be any triangle with vertices $\{a, b, c\}$, see Fig. 1 . For any $j, k \in$ $\{a, b, c\}$ let $\alpha_{j k} \in[0,2 \pi$ ) denote the argument of $j-k$ (in an arbitrarily fixed coordinate system for $\mathbb{R}^{2}$ ). Then, with the notation of Fig. 1, we have

$$
\alpha_{a c}=\alpha_{b a}+\theta_{a}+\pi \quad \bmod (2 \pi) ; \quad \alpha_{b c}=\alpha_{b a}-\theta_{b}+\pi \quad \bmod (2 \pi) .
$$

Remark (B). Note that $\mathcal{R}_{h}(\mathbf{z})$ is meaningful only when the points in the three-tuple $\mathbf{z}=\left\{z_{1}, z_{2}, z_{3}\right\}$ are non-collinear (so that $\operatorname{Area}(\Delta(\mathbf{z})) \neq 0$ ). However the set of noncollinear three-tuples in $\mathbb{C}$ viewed as a subset of $\mathbb{C}^{3}$ has full Lebesgue measure because the condition that $\operatorname{Area}(\Delta(\mathbf{z}))=0$ is equivalent to

$$
\vec{u} \times \vec{v}=\overrightarrow{0}
$$

with $\times$ denoting the cross product in $\mathbb{R}^{2}$ of the vectors $\vec{u}:=z_{2}-z_{1}$ and $\vec{v}:=z_{3}-z_{1}$. Since (4.3) is a quadratic equation in the variables $\left(z_{1}, z_{2}, z_{3}\right)$, its solution set has zero Lebesgue measure in $\mathbb{C}^{3}$ (it is an algebraic subvariety of $\mathbb{C}^{3}$ ); on the other hand, the product

$$
c^{2}(\mathbf{z}) \mathcal{R}_{h}(\mathbf{z})
$$

is meaningful as soon as the points $\left\{z_{1}, z_{2}, z_{3}\right\}$ are distinct from one another because, on account (3.14), it equals

$$
\frac{1}{\ell_{1} \ell_{2} \ell_{3}} \times(\text { a continuous function of } \mathbf{z}),
$$

and $\ell_{j} \neq 0$ for each $j=1,2,3$ (since the $z_{j}$ 's are distinct).

\subsection{Proof of Theorem 2.3}

Note that the implication $(i i) \Rightarrow$ ( $i$ ) is trivial, thus it suffices to show that

$$
\text { (iii) } \Rightarrow \text { (ii) and (i) } \Rightarrow \text { (iii). }
$$

We begin by proving that $(i i i) \Rightarrow(i i)$. We claim that

$$
\text { (iii) } \Rightarrow c^{2}(\mathbf{z}) \mathcal{R}_{h}(\mathbf{z})=0[\text { which immediately implies (ii) }] \text {. }
$$

To see this, let $c_{0}$ denote the assumed constant value of $h(z)$; we use the short-hand notation

$$
\alpha=2 c_{0}-2 \alpha_{21}
$$


[note that $\alpha=\alpha\left(z_{1}, z_{2}\right)$ because $\alpha_{21}$ is a function of $z_{1}$ and $z_{2}$ ]. Combining Proposition 2.2 the basic identity (3.12) we see that

$$
c^{2}(\mathbf{z}) \mathcal{R}_{h}(\mathbf{z})=\frac{1}{\ell_{2} \ell_{3}} \cos \left(\alpha-\theta_{1}\right)+\frac{1}{\ell_{1} \ell_{3}} \cos \left(\alpha+\theta_{2}\right)-\frac{1}{\ell_{1} \ell_{2}} \cos \left(\alpha+\theta_{2}-\theta_{1}\right)
$$

and thus

$$
c^{2}(\mathbf{z}) \mathcal{R}_{h}(\mathbf{z})=C(\mathbf{z}) \cos \alpha+D(\mathbf{z}) \sin \alpha,
$$

where

$$
C(\mathbf{z}):=\frac{\cos \theta_{1}}{\ell_{2} \ell_{3}}+\frac{\cos \theta_{2}}{\ell_{1} \ell_{3}}-\frac{1}{\ell_{1} \ell_{2}} \cos \left(\theta_{2}-\theta_{1}\right)
$$

and

$$
D(\mathbf{z}):=\frac{\sin \theta_{1}}{\ell_{2} \ell_{3}}-\frac{\sin \theta_{2}}{\ell_{1} \ell_{3}}+\frac{1}{\ell_{1} \ell_{2}} \sin \left(\theta_{2}-\theta_{1}\right) .
$$

We claim that

$$
C(\mathbf{z})=0, \quad \text { and } \quad D(\mathbf{z})=0
$$

Indeed, it follows from (3.12), (3.9) and the identity $\cos \theta_{3}=-\cos \left(\theta_{1}+\theta_{2}\right)$, that

$$
\begin{aligned}
C(\mathbf{z}) & =\frac{1}{2} c^{2}(\mathbf{z})-\left(\frac{\cos \theta_{3}+\cos \left(\theta_{2}-\theta_{1}\right)}{\ell_{1} \ell_{2}}\right) \\
& =\frac{1}{2} c^{2}(\mathbf{z})-2 \frac{\sin \theta_{1}}{\ell_{1}} \frac{\sin \theta_{2}}{\ell_{2}}=\frac{1}{2} c^{2}(\mathbf{z})-\frac{1}{2} c^{2}(\mathbf{z})=0 .
\end{aligned}
$$

To deal with $D(\mathbf{z})$ we invoke (3.10) and the identity $\sin \theta_{3}=\sin \left(\theta_{1}+\theta_{2}\right)$, which lead to

$$
D(\mathbf{z})=\frac{1}{\ell_{1} \ell_{2} \sin \theta_{3}}\left(\sin ^{2} \theta_{1}-\sin ^{2} \theta_{2}+\sin \left(\theta_{2}+\theta_{1}\right) \sin \left(\theta_{2}-\theta_{1}\right)\right)=0 .
$$

The proof of the implication: ( $i i i) \Rightarrow$ ( $i i)$ is concluded.

Next we prove that $(i) \Rightarrow$ ( $i i i)$. We show that if $h$ is continuous and non-constant then the inequality

$$
\left|\mathcal{R}_{h}(\mathbf{z})\right| \leq C \text { for any non-collinear three-tuple } \mathbf{z}
$$

is impossible. Fix a three-tuple $\mathbf{z}=\left\{0, z_{2}, z_{3}\right\}$ of non-collinear points with the property that the triangle $\Delta\left(0, z_{2}, z_{3}\right)$ has an obtuse angle at $z_{3}$, see Fig. 1 . Let $z_{4}$ denote the orthogonal projection of $z_{3}$ onto the opposite side of $\Delta\left(0, z_{2}, z_{3}\right)$, thus $z_{4}=\beta z_{2}$ with 
Fig. 3 Proof of Theorem 2.3: the triangles $\Delta\left(0, z_{2}, z_{3}\right)$ and $\Delta\left(0, z_{2}, z_{3}(\theta)\right)$

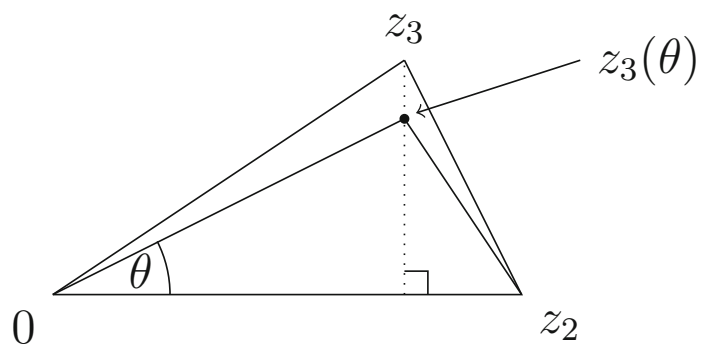

$0<\beta<1$. Consider the family of triangles $\Delta\left(0, z_{2}, z_{3}(\theta)\right)$ where $z_{3}(\theta)$ lies along the line segment whose endpoints are $z_{3}$ and $z_{4}$, see Fig. 3 .

Note that the three-tuples $\left(0, z_{2}, z_{3}\right)$ and $\left(0, z_{2}, z_{3}(\theta)\right)$ are arranged in admissible order. Thus, adopting the notation $\ell_{1}(\theta)$ and $\ell_{2}(\theta)$ for the lengths of the sides of $\Delta\left(0, z_{2}, z_{3}(\theta)\right)$ opposite to 0 and $z_{2}$, respectively, along with $\theta_{2}(\theta)$ for the angle at $z_{2}$, we may express $\mathcal{R}_{h}\left(0, z_{2}, z_{3}(\theta)\right)$ as the quotient

$$
\mathcal{R}_{h}\left(0, z_{2}, z_{3}(\theta)\right)=\frac{E\left(0, z_{2}, z_{3}(\theta)\right)}{F\left(0, z_{2}, z_{3}(\theta)\right)}
$$

with

$$
\begin{aligned}
E & \left(0, z_{2}, z_{3}(\theta)\right) \\
= & \ell_{1}(\theta) \cos \left(\mathfrak{h}_{0, z_{2}}(0)-\theta\right)+\ell_{2}(\theta) \cos \left(\mathfrak{h}_{0, z_{2}}\left(z_{2}\right)+\theta_{2}(\theta)\right) \\
& -\ell_{3} \cos \left(\mathfrak{h}_{0, z_{2}}\left(z_{3}(\theta)\right)+\theta_{2}(\theta)-\theta\right)
\end{aligned}
$$

and

$$
F\left(0, z_{2}, z_{3}(\theta)\right)=4 \ell_{3} \sin \theta \sin \theta_{2}(\theta),
$$

where the denominator $F\left(0, z_{2}, z_{3}(\theta)\right)$ comes from the identity

$$
\frac{\ell_{1}(\theta) \ell_{2}(\theta) \ell_{3}}{\left(4 \text { Area } \Delta\left(0, z_{2}, z_{3}(\theta)\right)\right)^{2}}=\frac{1}{4 \ell_{3} \sin \theta \sin \theta_{2}(\theta)}
$$

which in turn follows from (3.13) and (3.9).

Let $\omega$ denote the direction of the vector $0 \vec{z}_{2}$ that is, $\arg (\omega)=\alpha_{21}$. Now letting $\theta \rightarrow 0$, we have that: $z_{3}(\theta) \rightarrow z_{4}=\beta z_{2} ; \theta_{2}(\theta) \rightarrow 0 ; \ell_{2}(\theta) \rightarrow \beta \ell_{3}$, and $\ell_{1}(\theta) \rightarrow(1-\beta) \ell_{3}$. By the continuity of $h(z)$ we also have that $\mathfrak{h}_{0, z_{2}}\left(z_{3}(\theta)\right) \rightarrow \mathfrak{h}_{0, z_{2}}\left(z_{4}\right)=\mathfrak{h}_{0, z_{2}}\left(\beta z_{2}\right)$. Inserting these into (4.6) and (4.7) we obtain that

$E\left(0, z_{2}, z_{3}(\theta)\right) \rightarrow \ell_{3}\left[(1-\beta) \cos \left(\mathfrak{h}_{0, z_{2}}(0)\right)+\beta \cos \left(\mathfrak{h}_{0, z_{2}}\left(z_{2}\right)\right)-\cos \left(\mathfrak{h}_{0, z_{2}}\left(\beta z_{2}\right)\right)\right]$,

whereas

$$
F\left(0, z_{2}, z_{3}(\theta)\right) \rightarrow \ell_{3} \sin ^{2} 0=0
$$


Thus, by the assumed boundedness of $\mathcal{R}_{h}$ (that is condition (i ) ) we must have that

$$
\cos \left(\mathfrak{h}_{0, z_{2}}\left(\beta z_{2}\right)\right)=(1-\beta) \cos \left(\mathfrak{h}_{0, z_{2}}(0)\right)+\beta \cos \left(\mathfrak{h}_{0, z_{2}}\left(z_{2}\right)\right)
$$

for any $0<\beta<1$, for any direction $\omega$ and for any $z_{2}=\left|z_{2}\right| \omega$.

Before proceeding any further, we recall the definition of $\mathfrak{h}_{0, z_{2}}(z)$ :

$$
\mathfrak{h}_{0, z_{2}}(z)=2 h(z)-2 \arg z_{2}
$$

thus in particular we have that

$$
\mathfrak{h}_{0, \omega}(\zeta)=\mathfrak{h}_{0, t \omega}(\zeta) \text { for any direction } \omega, \quad \text { any } t>0 \text { and any } \zeta \in \mathbb{C} \text {. }
$$

Given the direction $\omega$ as above, let us consider any point $z$ in the direction of $\omega$, that is $z=|z| \omega$. We distinguish two cases:

Case 1. $|z|<1$ : Applying (4.8) to $z_{2}:=\omega$ and $\beta=|z|$ we find that

$$
\cos \left(\mathfrak{h}_{0, \omega}(z)\right)=(1-|z|) \cos \left(\mathfrak{h}_{0, \omega}(0)\right)+|z| \cos \left(\mathfrak{h}_{0, \omega}(\omega)\right)
$$

for any direction $\omega$ and for any $z=|z| \omega$ with $|z| \leq 1$.

Case 2. $|z|>1$ : In this case we apply (4.8) to $z_{2}:=z=|z| \omega$ and $\beta=1 /|z|$. Taking (4.9) into account it is easy to see that (4.10) also holds for $|z|>1$.

By continuity it follows that (4.10) must hold also for $|z|=1$. We conclude that the identity

$$
\cos \left(\mathfrak{h}_{0, \omega}(z)\right)=\cos \left(\mathfrak{h}_{0, \omega}(0)\right)+|z|\left(\cos \left(\mathfrak{h}_{0, \omega}(\omega)\right)-\cos \left(\mathfrak{h}_{0, \omega}(0)\right)\right)
$$

holds for any direction $\omega$ and for any $z=|z| \omega$. However note that the left-hand side of (4.11) is $O(1)$, while the right-hand side is $C_{\omega}+O(|z|)$, thus the only possibility is that

$$
\cos \left(\mathfrak{h}_{0, \omega}(\omega)\right)=\cos \left(\mathfrak{h}_{0, \omega}(0)\right) \equiv C_{\omega} \quad \text { for any } \quad|\omega|=1
$$

Substituting the latter into (4.11) we obtain

$$
\cos \left(\mathfrak{h}_{0, \omega}(z)\right)=C_{\omega} \text { for any }|\omega|=1 \text { and for any } z=|z| \omega \text {. }
$$

But recall that $\mathfrak{h}_{0, \omega}(z)=2 h(z)-2 \arg (\omega)$; thus it follows from (4.12) that

$$
h(z)=\arg (\omega)+\widetilde{C}_{\omega} \text { for any }|\omega|=1 \text { and for any } z=|z| \omega,
$$

which implies that

$$
h(z)=f\left(\frac{z}{|z|}\right), \quad z \in \mathbb{C} \backslash\{0\} .
$$


From this we conclude that $f$, and thus $h$, must be constant: if not, there would be two directions $\omega_{1} \neq \omega_{2}$ such that $f\left(\omega_{1}\right) \neq f\left(\omega_{2}\right)$. By the assumed continuity of $h(z)$ it would then follow that

$$
h(0)=\lim _{r \rightarrow 0} h\left(r \omega_{1}\right)=f\left(\omega_{1}\right) \neq f\left(\omega_{2}\right)=\lim _{r \rightarrow 0} h\left(r \omega_{2}\right)=h(0),
$$

which is a contradiction. The proof of $(i) \Rightarrow(i i i)$ is concluded, and so is the proof of [12, Theorem 5.3].

Remark (C). It is possible that the hypothesis that $h: \mathbb{C} \rightarrow \mathbb{R}$ is continuous may be relaxed. Below we give an example where $h(z)$ is constant except at one point and we show that the corresponding $\mathcal{R}_{h}$ is unbounded.

Fix $\epsilon_{0}>0$ such that $\sin \epsilon_{0}>1 / 2$ and set

$$
h(z)=\left\{\begin{array}{l}
0 \text { if } z \neq 0 \\
\frac{\epsilon_{0}}{2} \text { if } z=0 .
\end{array}\right.
$$

Consider three-tuples of the form $\mathbf{z}_{\lambda}=\{0,1, i \lambda\}$ with $\lambda>0$. Then it is easy to see that

$$
\mathcal{R}_{h}\left(\mathbf{z}_{\lambda}\right)=\frac{1+\lambda^{2}}{\lambda} \sin \epsilon_{0} .
$$

Now the condition $\left|\mathcal{R}_{h}(\mathbf{z})\right| \leq C$ for all three-tuples of non-collinear points would, in particular, require that

$$
\frac{1}{2}<\sin \epsilon_{0} \leq \frac{\lambda}{1+\lambda^{2}} C \text { for any } \lambda>0,
$$

which is not possible.

\subsection{Proof of Corollary 2.4}

If $h$ is constant then by Theorem 2.3 we have that $\mathcal{R}_{h}(\mathbf{z})=0$ for all three-tuples of non-collinear distinct points, thus

$$
\left(\frac{1}{2}-\mathcal{R}_{h}(\mathbf{z})\right)\left(\frac{1}{2}+\mathcal{R}_{h}(\mathbf{z})\right)=\frac{1}{4}>0 .
$$

Conversely, if

$$
\left(\frac{1}{2}-\mathcal{R}_{h}(\mathbf{z})\right)\left(\frac{1}{2}+\mathcal{R}_{h}(\mathbf{z})\right)>0
$$

for all three-tuples of non-collinear points then

$$
\left|\mathcal{R}_{h}(\mathbf{z})\right|<\frac{1}{2}
$$


and Theorem 2.3 gives that $h$ is constant.

\subsection{Proof of Theorem 2.5}

Conclusion (a) is immediate from Theorem 2.3 , thus we only need to prove conclusion (b) . Recall the definition $\mathfrak{h}_{z_{1}, z_{2}}(z):=2 h(z)-2 \operatorname{Arg}\left(z_{2}-z_{1}\right)$ (for an arbitrarily fixed coordinate system).

Lemma 4.2 Suppose that $h: \mathbb{C} \rightarrow \mathbb{R}$ is continuous and non-constant. Then there exist $z_{1} \neq z_{2}$ and $t_{0} \in(0,1)$ such that for $z_{0}=t_{0} z_{2}+\left(1-t_{0}\right) z_{1}$ we have

$$
\cos \mathfrak{h}_{z_{1}, z_{2}}\left(z_{1}\right)-\cos \mathfrak{h}_{z_{1}, z_{2}}\left(z_{0}\right)<0<\cos \mathfrak{h}_{z_{1}, z_{2}}\left(z_{2}\right)-\cos \mathfrak{h}_{z_{1}, z_{2}}\left(z_{0}\right)
$$

Proof It suffices to find $z_{1} \neq z_{2}$ such that

$$
\cos \mathfrak{h}_{z_{1}, z_{2}}\left(z_{1}\right)<\cos \mathfrak{h}_{z_{1}, z_{2}}\left(z_{2}\right)
$$

The existence of $z_{0}$ will then then follow by the mean-value theorem applied to $f(t):=$ $\left.\cos \mathfrak{h}_{z_{1}, z_{2}}\left(t z_{2}+(1-t) z_{1}\right)\right)$. We proceed by contradiction and suppose that

$$
\begin{aligned}
& \cos \mathfrak{h}_{z_{1}, z_{2}}\left(z_{1}\right)=\cos \mathfrak{h}_{z_{1}, z_{2}}\left(z_{2}\right) \text { for all } z_{1} \neq z_{2} \text {, that is, } \\
& \cos \left(2 h\left(z_{1}\right)-2 \operatorname{Arg}\left(z_{2}-z_{1}\right)\right)=\cos \left(2 h\left(z_{2}\right)-2 \operatorname{Arg}\left(z_{2}-z_{1}\right)\right) \text { for all } z_{1} \neq z_{2}
\end{aligned}
$$

But $\cos A=\cos B$ if and only if either $B-A \in 2 \pi \mathbb{Z}$ or $B+A \in 2 \pi \mathbb{Z}$. Thus for any $z_{1} \neq z_{2}$, there exists $k=k\left(z_{1}, z_{2}\right) \in \mathbb{Z}$ such that one of the two possibilities holds:

$$
2 h\left(z_{2}\right)-2 \operatorname{Arg}\left(z_{2}-z_{1}\right)=\left\{\begin{array}{l}
2 h\left(z_{1}\right)-2 \operatorname{Arg}\left(z_{2}-z_{1}\right)+2 k \pi, \text { or } \\
2 k \pi-2 h\left(z_{1}\right)+2 \operatorname{Arg}\left(z_{2}-z_{1}\right)
\end{array}\right.
$$

Equivalently stated,

$$
h\left(z_{2}\right) \in\left\{h\left(z_{1}\right)+k \pi: k \in \mathbb{Z}\right\} \cup\left\{k \pi-h\left(z_{1}\right)+2 \operatorname{Arg}\left(z_{2}-z_{1}\right): k \in \mathbb{Z}\right\} .
$$

If $h$ is continuous and non-constant, there exist $z_{0}, \omega \in \mathbb{C}$ and $|\omega|=1$ such that the map

$$
t \in \mathbb{R} \mapsto h\left(z_{0}+t \omega\right) \text { is continuous and non-constant. }
$$

Hence, the image set $h(\mathscr{L})$ contains an interval, where $\mathscr{L}=\left\{z_{0}+t \omega: t \in \mathbb{R}\right\}$. However setting $z_{1}=z_{0}$ and $z_{2} \in \mathscr{L}$ in (4.17), we find that

$$
h(\mathscr{L}) \subseteq\left\{h\left(z_{0}\right)+k \pi: k \in \mathbb{Z}\right\} \cup\left\{k \pi-h\left(z_{0}\right)+2 \omega: k \in \mathbb{Z}\right\}
$$

The right hand side above is a discrete set, whereas the left contains an interval, providing the desired contradiction. 


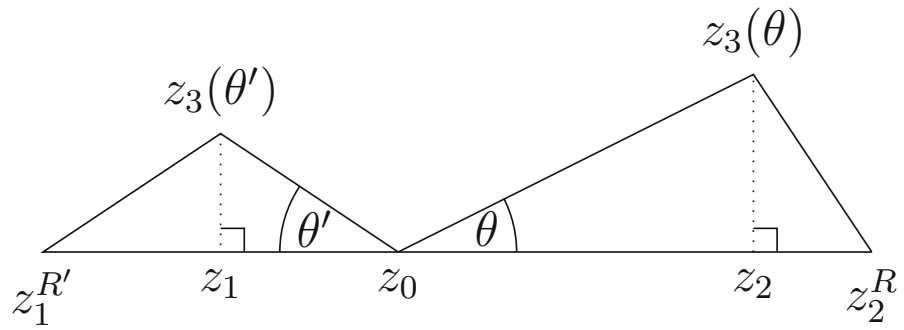

Fig. 4 Proof of Theorem 2.5: the families $\left\{\mathbf{z}_{R}(\theta)\right\}_{\theta, R}$ and $\left\{\mathbf{z}_{R^{\prime}}\left(\theta^{\prime}\right)\right\}_{\theta^{\prime}, R^{\prime}}$

Proof of ( $\mathrm{b}$ ). Let $z_{1}, z_{0}$ and $z_{2}$ be as in Lemma 4.2. Consider two families of noncollinear three-tuples $\left\{\mathbf{z}_{R}(\theta)\right\}_{\theta, R}$ and $\left\{\mathbf{z}_{R^{\prime}}\left(\theta^{\prime}\right)\right\}_{\theta^{\prime}, R^{\prime}}$ defined as follows:

$$
\mathbf{z}_{R}(\theta):=\left(z_{0}, z_{2}^{R}, z_{3}(\theta)\right), \quad \theta \in\left(0, \frac{\pi}{2}\right), \quad R>0
$$

where

$$
z_{2}^{R}:=\left(z_{2}-z_{0}\right)(1+R)+z_{0}
$$

and the point $z_{3}(\theta)$ has been chosen so that $\lim _{\theta \rightarrow 0} z_{3}(\theta)=z_{2}$, see Fig. 4; and

$$
\mathbf{z}_{R}\left(\theta^{\prime}\right):=\left(z_{1}^{R^{\prime}}, z_{0}, z_{2}^{R}, z_{3}\left(\theta^{\prime}\right)\right), \quad \theta^{\prime} \in\left(0, \frac{\pi}{2}\right), \quad R^{\prime}>0,
$$

where

$$
z_{1}^{R^{\prime}}:=R^{\prime}\left(z_{1}-z_{0}\right)+z_{1}
$$

and the point $z_{3}\left(\theta^{\prime}\right)$ has been chosen so that $\lim _{\theta^{\prime} \rightarrow 0} z_{3}\left(\theta^{\prime}\right)=z_{1}$, see again Fig. 4 .

Note that the three-tuples $\left(z_{0}, z_{2}^{R}, z_{3}(\theta)\right)$ and $\left(z_{1}^{R^{\prime}}, z_{0}, z_{3}\left(\theta^{\prime}\right)\right)$ are arranged in admissible order. We claim that there exist small $\theta_{0}$ and $\theta_{0}^{\prime}$, and large $R$ and $R^{\prime}$ such that

$$
\frac{1}{2}+\mathcal{R}_{h}\left(\mathbf{z}_{R_{0}}\left(\theta_{0}\right)\right)<0
$$

and

$$
\frac{1}{2}+\mathcal{R}_{h}\left(\mathbf{z}_{R_{0}^{\prime}}\left(\theta_{0}^{\prime}\right)\right)>0 .
$$

To prove claim (4.20) define

$$
\mathfrak{h}(z):=\mathfrak{h}_{z_{1}, z_{2}}(z), \quad z \in \mathbb{C}
$$

We point out that since $\mathfrak{h}_{z_{1}, z_{2}}(z)$ depends on $z_{1}$ and $z_{2}$ only through $\operatorname{Arg}\left(z_{2}-z_{1}\right)$ it follows that

$$
\mathfrak{h}_{z_{1}, z_{2}}(z)=\mathfrak{h}_{\tilde{z}_{1}, \tilde{z}_{2}}(z)
$$


whenever

$$
\operatorname{Arg}\left(z_{2}-z_{1}\right)=\operatorname{Arg}\left(\tilde{z}_{2}-\tilde{z}_{1}\right)
$$

In particular we have that

$$
\mathfrak{h}(z)=\mathfrak{h}_{z_{0}, z_{2}^{R}}(z)=\mathfrak{h}_{z_{1}^{R}, z_{0}}(z) .
$$

Invoking (2.6) to compute $1 / 2+\mathcal{R}_{h}\left(\mathbf{z}_{R}(\theta)\right)$ and using notation analogous to (4.5) we see that

$\operatorname{sign}\left(\frac{1}{2}+\mathcal{R}_{h}\left(\mathbf{z}_{R}(\theta)\right)\right)=\operatorname{sign}\left(F\left(\mathbf{z}_{R}(\theta)\right)+2 E\left(\mathbf{z}_{R}(\theta)\right)\right)$ for all $\theta>0, \quad R>0$,

where

$$
\begin{aligned}
F\left(\mathbf{z}_{R}(\theta)\right)= & 4(1+R)\left|z_{2}-z_{0}\right| \sin \theta \sin \theta_{2}(\theta, R), \text { and } \\
E\left(\mathbf{z}_{R}(\theta)\right)= & \ell_{1}^{R}(\theta) \cos \left(\mathfrak{h}\left(z_{0}\right)-\theta\right)+\ell_{2}(\theta) \cos \left(\mathfrak{h}\left(z_{2}^{R}\right)+\theta_{2}(\theta, R)\right) \\
& -\left|z_{2}-z_{0}\right|(1+R) \cos \left(\mathfrak{h}\left(z_{3}(\theta)+\theta_{2}(\theta, R)-\theta\right) .\right.
\end{aligned}
$$

We see that, for fixed $R>0$,

$$
\begin{aligned}
& \lim _{\theta \rightarrow 0}\left(F\left(\mathbf{z}_{R}(\theta)\right)+2 E\left(\mathbf{z}_{R}(\theta)\right)\right) \\
& \quad=2\left|z_{2}-z_{0}\right|\left(R\left(\cos \mathfrak{h}\left(z_{0}\right)-\cos \mathfrak{h}\left(z_{2}\right)\right)+\cos \mathfrak{h}\left(z_{2}^{R}\right)-\cos \mathfrak{h}\left(z_{2}\right)\right) .
\end{aligned}
$$

But (4.15) tells us that the latter is negative for sufficiently large $R$. Thus, by continuity, there are $\theta_{0} \ll 1$ and $R_{0} \gg 1$ such that (4.20) holds.

The proof of claim (4.21) is similar: proceeding as above we find that

$\operatorname{sign}\left(\frac{1}{2}+\mathcal{R}_{h}\left(\mathbf{z}_{R}\left(\theta^{\prime}\right)\right)\right)=\operatorname{sign}\left(F\left(\mathbf{z}_{R}\left(\theta^{\prime}\right)\right)+2 E\left(\mathbf{z}_{R}\left(\theta^{\prime}\right)\right)\right)$ for all $\theta>0, \quad R>0$,

where

$$
\begin{aligned}
F\left(\mathbf{z}_{R}\left(\theta^{\prime}\right)\right)= & 4(1+R)\left|z_{1}-z_{0}\right| \sin \theta^{\prime} \sin \theta_{1}\left(\theta^{\prime}, R\right), \text { and } \\
E\left(\mathbf{z}_{R}\left(\theta^{\prime}\right)\right)= & \ell_{1}\left(\theta^{\prime}\right) \cos \left(\mathfrak{h}\left(z_{1}^{R}\right)-\theta_{1}\left(\theta^{\prime}, R\right)\right)+\ell_{2}^{R}\left(\theta^{\prime}\right) \cos \left(\mathfrak{h}\left(z_{0}\right)+\theta^{\prime}\right) \\
& -\left|z_{1}-z_{0}\right|(1+R) \cos \left(\mathfrak{h}\left(z_{3}\left(\theta^{\prime}\right)+\theta^{\prime}-\theta_{1}\left(\theta^{\prime}, R\right)\right) .\right.
\end{aligned}
$$

We obtain (again for fixed $R>0$ )

$$
\begin{aligned}
& \lim _{\theta^{\prime} \rightarrow 0}\left(F\left(\mathbf{z}_{R}\left(\theta^{\prime}\right)\right)+2 E\left(\mathbf{z}_{R}(\theta)\right)\right) \\
& \quad=2\left|z_{1}-z_{0}\right|\left(R\left(\cos \mathfrak{h}\left(z_{0}\right)-\cos \mathfrak{h}\left(z_{1}\right)\right)+\cos \mathfrak{h}\left(z_{1}^{R}\right)-\cos \mathfrak{h}\left(z_{1}\right)\right),
\end{aligned}
$$


which is positive by (4.15), for $R$ large enough. Therefore, by continuity, there are $\theta_{0}^{\prime} \ll 1$ and $R_{0}^{\prime} \gg 1$ such that

$$
\frac{1}{2}+\mathcal{R}_{h}\left(\mathbf{z}_{R_{0}^{\prime}}\left(\theta_{0}^{\prime}\right)\right)>0
$$

The proof of Theorem 2.5 is concluded.

\subsection{Proof of Proposition 2.7}

On account of (3.7) and (3.3) we have

$$
S\left[K_{h}^{*}\right](\mathbf{z})=\frac{2}{\ell_{1}^{2} \ell_{2}^{2} \ell_{3}^{2}} \sum_{\substack{j \\ k<l}} \ell_{j}^{2} \operatorname{Re}\left(\mathrm{e}^{-i\left(h\left(z_{k}\right)-h\left(z_{l}\right)\right)}\left(z_{k}-z_{j}\right)\left(\overline{z_{l}-z_{j}}\right)\right) .
$$

Adopting the labeling scheme for $\Delta(\mathbf{z})$ that was described in Sect. 3.3, we expand the above sum and invoke (3.15) and (3.16) to express each of $\left(z_{k}-z_{j}\right)$, resp. $\left(\overline{z_{l}-z_{j}}\right)$, in terms of $\left(z_{2}-z_{1}\right)$, resp. $\left(\overline{z_{2}-z_{1}}\right)$. This leads us to the identity

$$
\begin{aligned}
S\left[K_{h}^{*}\right](\mathbf{z})= & \frac{2}{\ell_{1}^{2} \ell_{2}^{2} \ell_{3}^{2}}\left[\ell_{1}^{2} \ell_{3}^{2} \frac{\beta}{\cos \theta_{1}} \cos \left(h\left(z_{2}\right)-h\left(z_{3}\right)+\theta_{1}\right)\right. \\
& +\ell_{2}^{2} \ell_{3}^{2} \frac{(1-\beta)}{\cos \theta_{2}} \cos \left(h\left(z_{1}\right)-h\left(z_{3}\right)-\theta_{2}\right) \\
& \left.-\ell_{3}^{4} \frac{\beta}{\cos \theta_{1}} \frac{(1-\beta)}{\cos \theta_{2}} \cos \left(h\left(z_{1}\right)-h\left(z_{2}\right)-\left(\theta_{1}+\theta_{2}\right)\right)\right] .
\end{aligned}
$$

The latter simplifies to

$$
\begin{aligned}
S\left[K_{h}^{*}\right](\mathbf{z})= & \frac{2}{\ell_{1} \ell_{2} \ell_{3}}\left[\ell_{1} \ell_{3} \frac{\beta}{\ell_{2} \cos \theta_{1}} \cos \left(h\left(z_{2}\right)-h\left(z_{3}\right)+\theta_{1}\right)\right. \\
& +\ell_{2} \ell_{3} \frac{(1-\beta)}{\ell_{1} \cos \theta_{2}} \cos \left(h\left(z_{1}\right)-h\left(z_{3}\right)-\theta_{2}\right) \\
& \left.-\ell_{3}^{3} \frac{\beta}{\ell_{2} \cos \theta_{1}} \frac{(1-\beta)}{\ell_{1} \cos \theta_{2}} \cos \left(h\left(z_{1}\right)-h\left(z_{2}\right)-\left(\theta_{1}+\theta_{2}\right)\right)\right] .
\end{aligned}
$$

Recalling (3.14) and (3.17) we conclude that

$$
\mathrm{S}\left[K_{h}^{*}\right](\mathbf{z})=c^{2}(\mathbf{z}) \mathcal{H}(\mathbf{z})
$$

with $\mathcal{H}(\mathbf{z})$ as in (2.11). The proof is concluded.

We point out that the arguments in Remark (B) (showing the well posedness of the quantity $c^{2}(z) \mathcal{R}_{h}(\mathbf{z})$ for triples of distinct (but possibly collinear) points) apply verbatim to $c^{2}(\mathbf{z}) \mathcal{H}(\mathbf{z})$. 


\subsection{Proof of Theorem 2.8}

Since the implication ( $i \mathrm{i}) \Rightarrow$ ( $i$ ) is trivial, we only need to prove that

$$
\text { (iii) } \Rightarrow \text { (ii) and (i) } \Rightarrow \text { (iii). }
$$

We first show that $(i i i) \Rightarrow$ ( $i i)$. If $h(z)=$ const. then Proposition 2.7 gives that

$$
\mathcal{H}(\mathbf{z})=2 \frac{\ell_{1} \ell_{2} \ell_{3}}{(4 \operatorname{Area} \Delta(\mathbf{z}))^{2}}\left\{\ell_{1} \cos \theta_{1}+\ell_{2} \cos \theta_{2}+\ell_{3} \cos \theta_{3}\right\}
$$

and it follows from (3.14) and (3.12) that the latter equals 1.

We are left to prove the implication (i) $\Rightarrow$ (iii). Equivalently, we show that if $h$ is continuous and non-constant then the inequality

$$
|\mathcal{H}(\mathbf{z})| \leq C \text { for any non-collinear three-tuple } \mathbf{z}
$$

is impossible. To this end, first note that $S\left[K_{h}^{*}\right]=S\left[K_{h+c}^{*}\right]$, for any constant $c$. Thus we may assume without loss of generality that $h(0)=0$. Define

$$
\begin{aligned}
S & :=\{z \in \mathbb{C}: h(z) \notin 2 \pi \mathbb{Z}\}, \text { and } \\
\mathcal{A}_{z} & :=\left\{\cot \left(\frac{h(z)}{4}\right),-\tan \left(\frac{h(z)}{4}\right)\right\}, \quad \text { for } z \in S .
\end{aligned}
$$

Note that $h(z) \neq 0$ for each $z \in S$, and in particular $0 \notin S$.

Lemma 4.3 $S$ is nonempty, and hence $\mathcal{A}_{z}$ is well-defined for every $z \in S$.

Proof If $S=\emptyset$, this means either $h \equiv 0$, or that $h(\mathbb{C})$ is disconnected. The first case contradicts the fact that $h$ is nonconstant, and the second contradicts its continuity.

Lemma 4.4 For every $z_{0} \in S$ there exist two numbers $s \neq t \in \mathbb{R}^{+}$such that

$$
\begin{aligned}
& \left\{s z_{0}, t z_{0}\right\} \subseteq S, \text { and } \\
& \mathcal{A}_{s z_{0}} \cap \mathcal{A}_{t z_{0}}=\emptyset .
\end{aligned}
$$

Proof By continuity of $h$, the function $g: \mathbb{R} \rightarrow \mathbb{R}$ given by

$$
g(t):=h\left(t z_{0}\right)
$$

is a non-constant continuous function on $\mathbb{R}$, and we have that $g(0)=0, g(1) \neq 0$. The intermediate value theorem ensures that for any small $\epsilon>0$, there exists $t_{0} \in(0,1)$ such that $0<\left|g\left(t_{0}\right)\right|<\epsilon<2 \pi$. Let us choose $\epsilon>0$ small enough so that

$$
\begin{aligned}
& \alpha \mapsto \cot (\alpha / 4), \quad \alpha \mapsto-\tan (\alpha / 4) \text { are both injective on }|\alpha|<\epsilon, \text { and } \\
& \{\cot (\alpha / 4):|\alpha|<\epsilon\} \cap\{-\tan (\alpha / 4):|\alpha|<\epsilon\}=\emptyset
\end{aligned}
$$




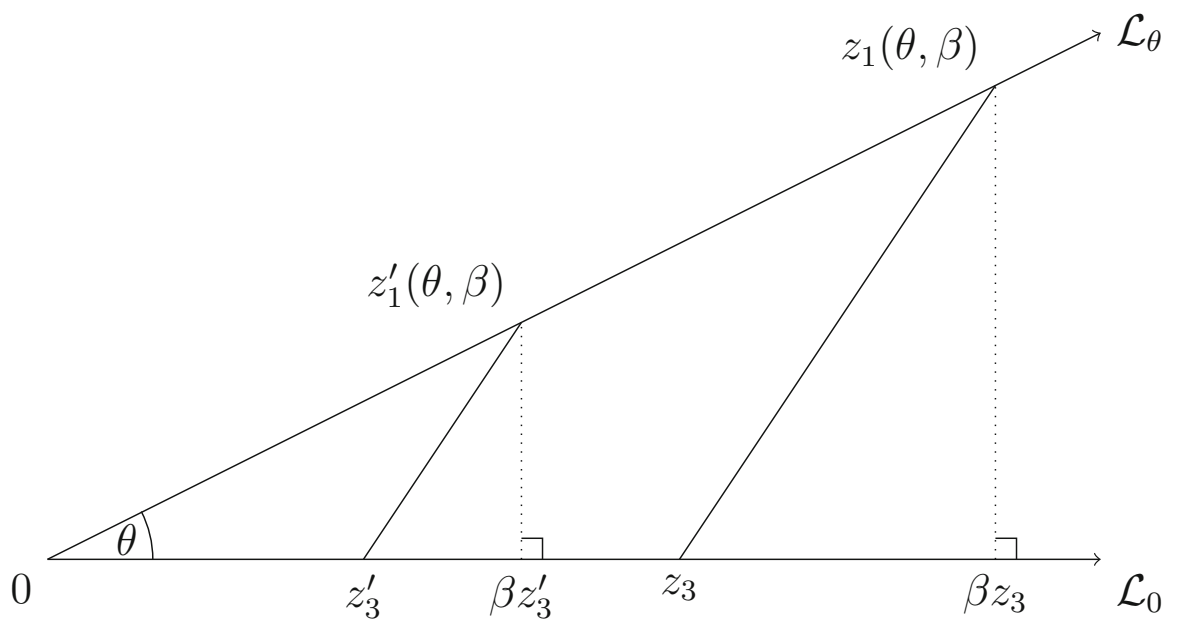

Fig. 5 Proof of Theorem 2.8: the three-tuples $\left(z_{1}^{\prime}(\theta, \beta), 0, z_{3}^{\prime}\right)$ and $\left(z_{1}(\theta, \beta), 0, z_{3}\right)$

Without loss of generality, assume $g\left(t_{0}\right)>0$. By the intermediate value theorem again, $g$ assumes every value between $g(0)=0$ and $g\left(t_{0}\right)$ on the interval $\left[0, t_{0}\right]$. In particular, let $s$ and $t$ denote two distinct points in $\left(0, t_{0}\right)$ such that $g(s) \neq g(t)$ and $g(s), g(t) \in\left(0, g\left(t_{0}\right)\right)$. The conclusions of the lemma then hold for this choice of $s$ and $t$.

We now continue with the proof of the implication $(i) \Rightarrow$ (iii) in Theorem 2.8. Let $0 \neq z_{0} \in S$ and $s \neq t \in \mathbb{R}^{+}$be as in Lemmas 4.3 and 4.4, respectively. Set

$$
z_{3}:=s z_{0} \in S \text { and } z_{3}^{\prime}:=t z_{0} \in S
$$

Thus, the points $0, z_{3}$ and $z_{3}^{\prime}$ are distinct but collinear, and they determine a ray $\mathcal{L}_{0}$ depicted in Fig. 5. Now let $\beta>1$ be given and define two points

$$
z_{4}(\beta):=\beta z_{3} \in \mathcal{L}_{0}, \quad \text { and } \quad z_{4}^{\prime}(\beta):=\beta z_{3}^{\prime} \in \mathcal{L}_{0}
$$

For any $\theta \in(0, \pi / 2)$ let $\mathcal{L}_{\theta}$ be (any fixed) ray forming an angle $\theta$ with $\mathcal{L}_{0}$, see Fig. 5 .

Next we let

$$
z_{1}(\theta, \beta) \in \mathcal{L}_{\theta} \quad \text { and } \quad z_{1}^{\prime}(\theta, \beta) \in \mathcal{L}_{\theta}
$$

be the intersection points with $\mathcal{L}_{\theta}$ of the lines perpendicular to $\mathcal{L}_{0}$ and passing through $z_{4}(\beta)$ and $z_{4}^{\prime}(\beta)$, respectively. It is easy to check that the three-tuples $\left(z_{1}(\theta, \beta), 0, z_{3}\right)$ and $\left(z_{1}^{\prime}(\theta, \beta), 0, z_{3}^{\prime}\right)$ are arranged in admissible form: see Fig. 5. 
Let us focus for a moment on the family of triangles determined by the first three-tuple, $\Delta\left(z_{1}(\theta, \beta), 0, z_{3}\right)$ : it follows from (2.11) that

$$
\frac{(4 \operatorname{Area}(\Delta))^{2} \mathcal{H}\left(z_{1}(\theta, \beta), 0, z_{3}\right)}{\ell_{1} \ell_{2}(\theta, \beta) \ell_{3}(\theta, \beta)}=A\left(\theta, \beta, z_{3}\right)
$$

holds for any $\beta>1$ and $0<\theta<\pi / 2$, where we have set

$$
\begin{aligned}
A\left(\theta, \beta, z_{3}\right):= & \ell_{1} \cos \left(h\left(z_{3}\right)+\theta\right)+\ell_{2}(\theta, \beta) \cos \left(h\left(z_{1}(\theta, \beta)-h\left(z_{3}\right)-\theta\right)\right. \\
& -\ell_{3}(\theta, \beta) \cos \left(h\left(z_{1}(\theta, \beta)\right)-\theta_{1}(\theta, \beta)-\theta\right) .
\end{aligned}
$$

(Recall that $h(0)=0$.) Next we point out that

$$
4 \operatorname{Area}(\Delta)=2 \ell_{1}^{2} \beta \tan \theta ; \ell_{2}(\theta, \beta)=\ell_{1}\left(\frac{\beta^{2}}{\cos ^{2} \theta}+(1-2 \beta)\right)^{\frac{1}{2}} \text {, and } \ell_{3}(\theta, \beta)=\ell_{1} \frac{\beta}{\cos \theta}
$$

Thus if (4.24) were to hold, we would have that

$$
\lim _{\theta \rightarrow 0} \frac{(4 \operatorname{Area}(\Delta))^{2} \mathcal{H}\left(z_{1}(\theta, \beta), 0, z_{3}\right)}{\ell_{1} \ell_{2}(\theta, \beta) \ell_{3}(\theta, \beta)}=0 \text { for all } \beta>1,
$$

and this, in turn, would imply that

$$
0=\lim _{\theta \rightarrow 0} A\left(\theta, \beta, z_{3}\right)=\ell_{1}\left(\cos \left(h\left(z_{3}\right)\right)+(\beta-1) \cos \left(h\left(\beta z_{3}\right)-h\left(z_{3}\right)\right)-\beta \cos \left(h\left(\beta z_{3}\right)\right)\right)
$$

for all $\beta>1$, that is

$$
\cos \left(h\left(z_{3}\right)\right)+(\beta-1) \cos \left(h\left(\beta z_{3}\right)-h\left(z_{3}\right)\right)-\beta \cos \left(h\left(\beta z_{3}\right)\right)=0 \text { for all } \beta>1
$$

Applying this same reasoning to the family of triangles $\Delta\left(z_{1}^{\prime}(\theta, \beta), 0, z_{3}^{\prime}\right)$, we similarly obtain that

$$
\cos \left(h\left(z_{3}^{\prime}\right)\right)+(\beta-1) \cos \left(h\left(\beta z_{3}^{\prime}\right)-h\left(z_{3}^{\prime}\right)\right)-\beta \cos \left(h\left(\beta z_{3}^{\prime}\right)\right)=0 \text { for all } \beta>1
$$

In summary: if (4.24) were to hold for all non-collinear three-point configurations, then (4.29) and (4.30) would have to be true for all $\beta>1$.

Applying the half-angle identities:

$$
\begin{gathered}
\sin (\alpha)=2 \sin \left(\frac{\alpha}{2}\right) \cos \left(\frac{\alpha}{2}\right) ; \cos (\alpha)=\cos ^{2}\left(\frac{\alpha}{2}\right)-\sin ^{2}\left(\frac{\alpha}{2}\right) \\
\text { to } \alpha:=h\left(\beta z_{3}\right)
\end{gathered}
$$

we see that (4.29) has the equivalent formulation 


$$
\begin{aligned}
& (1-\beta)\left\{\cos \left(h\left(z_{3}\right)\right)\left[\cos ^{2}\left(\frac{\alpha}{2}\right)-\sin ^{2}\left(\frac{\alpha}{2}\right)\right]\right. \\
& \left.+2 \sin \left(h\left(z_{3}\right)\right) \sin \left(\frac{\alpha}{2}\right) \cos \left(\frac{\alpha}{2}\right)\right\}+ \\
& +\beta\left[\cos ^{2}\left(\frac{\alpha}{2}\right)-\sin ^{2}\left(\frac{\alpha}{2}\right)\right]-\cos \left(h\left(z_{3}\right)\right)\left[\cos ^{2}\left(\frac{\alpha}{2}\right)+\sin ^{2}\left(\frac{\alpha}{2}\right)\right]=0 \text { for all } \beta>1 .
\end{aligned}
$$

A corresponding formulation holds for (4.30) (with $\alpha:=h\left(\beta z_{3}^{\prime}\right)$ ). There are now two possibilities:

Case 1: $\quad \beta z_{3} \in S$ and $\beta z_{3}^{\prime} \in S$ for all $\beta>1$.

Case 2: $\quad \beta_{0} z_{3} \notin S$ and/or $\beta_{0}^{\prime} z_{3}^{\prime} \notin S$ for some $\beta_{0}$ and/or $\beta_{0}^{\prime}>1$.

We show that each of Cases 1 and 2 is either impossible, or leads to a contradiction.

Analysis of Case 1: In this case we have that

$$
\sin \left(\frac{h\left(\beta z_{3}\right)}{2}\right) \neq 0 \text { and } \sin \left(\frac{h\left(\beta z_{3}^{\prime}\right)}{2}\right) \neq 0 \text { for all } \beta>1
$$

Thus (4.29) can be equivalently formulated as

$$
\beta\left(1-\cos \left(h\left(z_{3}\right)\right) Y_{\beta}^{2}+2(1-\beta) \sin \left(h\left(z_{3}\right)\right) Y_{\beta}-\left(\beta+(2-\beta) \cos \left(h\left(z_{3}\right)\right)=0\right.\right.
$$

for all $\beta>1$, where we have set

$$
Y_{\beta}:=\cot \left(\frac{h\left(\beta z_{3}\right)}{2}\right) .
$$

Similarly, we have that (4.30) is restated as

$$
\beta\left(1-\cos \left(h\left(z_{3}^{\prime}\right)\right) Z_{\beta}^{2}+2(1-\beta) \sin \left(h\left(z_{3}^{\prime}\right)\right) Z_{\beta}-\left(\beta+(2-\beta) \cos \left(h\left(z_{3}^{\prime}\right)\right)=0\right.\right.
$$

for all $\beta>1$, with

$$
Z_{\beta}:=\cot \left(\frac{h\left(\beta z_{3}^{\prime}\right)}{2}\right) .
$$

Note that (4.32) is a quadratic equation with discriminant $D(\beta)$ that satisfies

$$
\frac{D(\beta)}{4}=2\left(1-\cos \left(h\left(z_{3}\right)\right) \beta^{2}-2\left(1-\cos \left(h\left(z_{3}\right)\right) \beta+\sin ^{2}\left(h\left(z_{3}\right)\right), \text { for all } \beta>1\right. \text {. }\right.
$$

Thus $D(\beta)$ lies on an up-ward looking parabola with vertex at $\beta_{0}=1 / 2$ and it follows that

$$
D(\beta)>4 \sin ^{2}\left(h\left(z_{3}\right)\right) \geq 0 \text { for all } \beta>1 .
$$


Similarly, the discriminant $D^{\prime}(\beta)$ of the quadratic equation (4.34) has

$$
D^{\prime}(\beta)>4 \sin ^{2}\left(h\left(z_{3}^{\prime}\right)\right) \geq 0 \text { for all } \beta>1 .
$$

It follows that each of (4.32) and (4.34) can be solved algebraically, giving us that

$$
\cot \left(\frac{h\left(\beta z_{3}\right)}{2}\right)
$$

equals one of the two quantities

$$
\frac{(\beta-1) \sin \left(h\left(z_{3}\right)\right) \pm\left\{(\beta-1)^{2} \sin ^{2}\left(h\left(z_{3}\right)\right)+\beta\left(1-\cos \left(h\left(z_{3}\right)\right)\left(\beta+(2-\beta) \cos \left(h\left(z_{3}\right)\right)\right)\right\}^{1 / 2}\right.}{\beta\left(1-\cos \left(h\left(z_{3}\right)\right)\right.}
$$

for any $\beta>1$. We now let $\beta \rightarrow \infty$. One can verify that quantities above converge as $\beta \rightarrow \infty$ with limits $\cot \left(\frac{h\left(z_{3}\right.}{4}\right) ;-\tan \left(\frac{h\left(z_{3}\right.}{4}\right)$ respectively for the " + " and " - " determinations.

We similarly have that $\cot \left(\frac{h\left(\beta z_{3}^{\prime}\right)}{2}\right)$ equals one of

$$
\frac{(\beta-1) \sin \left(h\left(z_{3}^{\prime}\right)\right) \pm\left\{(\beta-1)^{2} \sin ^{2}\left(h\left(z_{3}^{\prime}\right)\right)+\beta\left(1-\cos \left(h\left(z_{3}^{\prime}\right)\right)\left(\beta+(2-\beta) \cos \left(h\left(z_{3}^{\prime}\right)\right)\right)\right\}^{1 / 2}\right.}{\beta\left(1-\cos \left(h\left(z_{3}^{\prime}\right)\right)\right.},
$$

and the latter converge as $\beta \rightarrow \infty$ to

$$
\cot \left(\frac{h\left(z_{3}^{\prime}\right)}{4}\right) ;-\tan \left(\frac{h\left(z_{3}^{\prime}\right)}{4}\right) \text { respectively. }
$$

It follows that

$$
\lim _{\beta \rightarrow \infty} \cot \left(\frac{h\left(\beta z_{3}\right)}{2}\right) \in\left\{\cot \left(\frac{h\left(z_{3}\right)}{4}\right) ;-\tan \left(\frac{h\left(z_{3}\right)}{4}\right)\right\}
$$

and

$$
\lim _{\beta \rightarrow \infty} \cot \left(\frac{h\left(\beta z_{3}^{\prime}\right)}{2}\right) \in\left\{\cot \left(\frac{h\left(z_{3}^{\prime}\right)}{4}\right) ;-\tan \left(\frac{h\left(z_{3}^{\prime}\right)}{4}\right)\right\} .
$$

On the other hand, by our choice of $z_{3}:=s z_{0}$ and $z_{3}^{\prime}=t z_{0}$ with $s, t \in \mathbb{R}^{+}$we also have that

$$
\lim _{\beta \rightarrow \infty} \cot \left(\frac{h\left(\beta z_{3}\right)}{2}\right)=\lim _{\beta \rightarrow \infty} \cot \left(\frac{h\left(\beta z_{3}^{\prime}\right)}{2}\right)
$$


The latter gives us a contradiction because by Lemma 4.4 we have that

$$
\left\{\cot \left(\frac{h\left(z_{3}\right)}{4}\right) ;-\tan \left(\frac{h\left(z_{3}\right)}{4}\right)\right\} \cap\left\{\cot \left(\frac{h\left(z_{3}^{\prime}\right)}{4}\right) ;-\tan \left(\frac{h\left(z_{3}^{\prime}\right)}{4}\right)\right\}=\varnothing
$$

The analysis of Case 1 is concluded.

Analysis of Case 2: Suppose, for instance, that $\beta_{0} z_{3} \notin S$ for some $\beta_{0}>1$. Then (4.31) for $\beta=\beta_{0}$ reads

$$
\left(1-\beta_{0}\right) \cos \left(h\left(z_{3}\right)\right)+\beta_{0}-\cos \left(h\left(z_{3}\right)\right)=0
$$

giving us

$$
\cos \left(h\left(z_{3}\right)\right)=1, \quad \text { that is } z_{3} \notin S \text {. }
$$

But this is impossible because $z_{3} \in S$. The situation when $\beta_{0}^{\prime} z_{3}^{\prime} \notin S$ for some $\beta_{0}^{\prime}>1$ is treated in the same way.

The proof of Theorem 2.8 is concluded.

Remark (D). It is possible that the hypothesis that $h: \mathbb{C} \rightarrow \mathbb{R}$ is continuous may be relaxed. Below we give an example where $h(z)$ is constant except at one point and we show that the corresponding $\mathcal{H}$ is unbounded.

Fix $\epsilon_{0}>0$ such that $\sin \epsilon_{0}>1 / 2$ and set

$$
h(z)= \begin{cases}0 & \text { if } z \neq 0 \\ -\epsilon_{0} & \text { if } z=0 .\end{cases}
$$

Consider three-tuples of the form $\mathbf{z}_{\lambda}=\{0,1, i \lambda\}$ with $\lambda>0$. Then it is easy to see that

$$
\mathcal{H}\left(\mathbf{z}_{\lambda}\right)=\frac{1}{2} \frac{\left(1+\lambda^{2}\right)^{1 / 2}}{\lambda}\left\{\lambda \cos \left(\theta_{2, \lambda}+\epsilon_{0}\right)+\sin \left(\theta_{2, \lambda}+\epsilon_{0}\right)\right\}
$$

where $\theta_{2, \lambda}$ is the angle of $\Delta(0,1, i \lambda)$ at the vertex $z_{2}=1$.

Now the condition $|\mathcal{H}(\mathbf{z})| \leq C$ for all three-tuples of non-collinear points would, in particular, require that

$$
\left\{\lambda \cos \left(\theta_{2, \lambda}+\epsilon_{0}\right)+\sin \left(\theta_{2, \lambda}+\epsilon_{0}\right)\right\} \leq \frac{2 \lambda}{\left(1+\lambda^{2}\right)^{1 / 2}} C \text { for any } \lambda>0
$$

which is not possible because $\theta_{2, \lambda} \rightarrow 0$ as $\lambda \rightarrow 0$ and $\sin \epsilon_{0}>1 / 2$.

Acknowledgements Part of this work took place $(a)$ at the Banff International Research Station in Banff, Alberta during a weeklong "Research in Teams" Program in the summer of 2016, and $(b)$ at the Mathematical Sciences Research Institute in Berkeley, California, where the authors were in residence during a thematic program in the spring of 2017. We thank both institutes for their support and hospitality. We are most grateful to Karthik Ramaseshan for his assistance in generating all diagrams, and to the anonymous referees for their helpful feedback. 
Funding The authors were supported respectively by Award No. DMS-1503612 from the National Science Foundation USA and a Discovery Grant (EG 1508) from the National Science and Engineering Research Council of Canada.

\section{Declarations}

Conflict of interest The authors have no conflicts of interest to declare that are relevant to the content of this article.

\section{References}

1. Bell, S.R.: The Cauchy integral, the Szegö projection, the Dirichlet problem and the Ahlfors map. Contemp. Math. 137, 43-61 (1992)

2. Calderón, A.-P.: Cauchy integrals on Lipschitz curves and related operators. Proc. Natl Acad. Sci. USA 74(4), 1324-1327 (1977)

3. Chousionis, V., Mateu, J., Prat, L., Tolsa, X.: Calderòn-Zygmund kernels and rectifiability in the plane. Adv. Math. 231(2012), 535-568 (2012)

4. Chousionis, V., Mateu, J., Prat, L., Tolsa, X.: Capacities associated with Calderón-Zygmund kernels. J. Potential Anal. 38(3), 913-949 (2013)

5. Chousionis, V., Prat, L.: Some Calderòn-Zygmund kernels and their relation to Wolff capacity. Math. Z. 282, 435-460 (2016)

6. Chunaev, P.: A new family of singular integral operators whose $L^{2}$ boundedness implies rectifiability. J. Geom. Anal. 27, 2725-2757 (2017)

7. Chunaev, P., Mateu, J., Tolsa, X.: Singular integrals unsuitable for the curvature method whose $L^{2}$ boundedness still implies rectifiability. J. Anal. Math. 138, 741-764 (2019)

8. Chunaev, P., Mateu, J., Tolsa, X.: A family of singular integral operators which control the Cauchy transform. Math. Z. 294, 1283-1340 (2020)

9. Coifman, R., McIntosh, A., Meyer, Y.: Cauchy integrals on Lipschitz curves and related operators, Proc. at. Acad. Sci. U.S.A. 74 (1977), no. 4, 1324-1327. L' integrale de Cauchy definit un operateur bornè sur $L^{2}$ pour les courbes Lipschitziennes, Ann. Math. 116, 361-387 (1982)

10. David, G.: Analytic capacity, Cauchy kernel, Menger curvature, and rectifiability. In: Harmonic Analysis and Partial Differential Equations (Chicago, IL, 1996), Chicago Lectures in Mathematics, pp. 183-197. University Chicago Press, Chicago (1999)

11. Farag, H.: The Riesz kernels do not give rise to higher dimensional analogues of the Menger-Melnikov curvature. Publ. Mat. 43, 251-260 (1999)

12. Lanzani, L., Pramanik, M.: Symmetrization of a Cauchy-like kernel on curves. J. Funct. Anal. (Preprint). arXiv:2001.09375

13. Lerman, G., Whitehouse, T.: High-dimensional Menger-type curvatures-Part I: geometric multipoles and multiscale inequalities. Rev. Mat. Iberoam. 27(2), 493-555 (2011)

14. Lerman, G., Whitehouse, T.: High-dimensional Menger-type curvatures-Part II: $d$-separation and a menagerie of curvatures. Constr. Approx. 30, 325-360 (2009)

15. Mateu, J., Prat, L., Verdera, J.: The capacity associated to signed Riesz kernels, and Wolff potentials. J. Reine Angew. Math. 578, 201-223 (2005)

16. Mattila, P., Melnikov, M., Verdera, J.: The Cauchy integral, analytic capacity, and uniform rectifiability. Ann. Math. (2) 144(1), 127-136 (1996)

17. Melnikov, M.S.: Analytic capacity: a discrete approach and the curvature of measures. Mat. Sb. 186(6), 57-76 (1995)

18. Melnikov, M., Verdera, J.: A geometric proof of the $L^{2}$ boundedness of the Cauchy integral on Lipschitz graphs. Int. Math. Res. Not. 7, 325-331 (1995)

19. Pajot, H.: Analytic Capacity, Rectifiability, Menger Curvature and the Cauchy Integral. Lecture Notes in Mathematics, vol. 1799. Springer, Berlin (2002)

20. Prat, L.: Potential theory of signed Riesz kernels: capacity and Hausdorff measure. Int. Math. Res. Not. 19, 937-981 (2004) 
21. Stein, E.: Harmonic Analysis: Real-Variable Methods, Orthogonality and Oscillatory Integrals. Princeton Mathematical Series, 43, Monographs in Harmonic Analysis III, Princeton University Press, Princeton (1993)

22. Tolsa, X.: Analytic Capacity, the Cauchy Transform, and Non-homogeneous Calderón-Zygmund Theory. Progress in Mathematics, vol. 307. Birkhäuser, Boston (2014)

23. Verchota, G.: Layer potentials and regularity of the Dirichlet problem for Laplace's equation in Lipschitz domains. J. Funct. Anal. 59, 572-611 (1984)

24. Verdera, J.: $L^{2}$ boundedness of the Cauchy integral and Menger curvature. Contemp. Math. 277, 139-158 (2001)

Publisher's Note Springer Nature remains neutral with regard to jurisdictional claims in published maps and institutional affiliations. 